Article

\title{
Improving Breviscapine Oral Bioavailability by Preparing Nanosuspensions, Liposomes and Phospholipid Complexes
}

\author{
Zilin Song ${ }^{1}$, Jiaojiao Yin ${ }^{1}$, Peifu Xiao ${ }^{1}$, Jin Chen ${ }^{1}$, Jingxin Gou ${ }^{1}$, Yanjiao Wang ${ }^{1}$, Yu Zhang ${ }^{1}$, Tian Yin ${ }^{2}$, \\ Xing Tang ${ }^{1}$ and Haibing $\mathrm{He}^{1, *}$ \\ 1 Department of Pharmaceutics, College of Pharmacy, Shenyang Pharmaceutical University, Shenyang 110016, \\ China; songzilin93@163.com (Z.S.); Yinjiaojiao0406@163.com (J.Y.); xiaopeifu96@163.com (P.X.); \\ chenjin951102@126.com (J.C.); paladin.13@163.com (J.G.); wang53k2002@163.com (Y.W.); \\ zhangyu@syphu.edu.cn (Y.Z.); tanglab@126.com (X.T.) \\ 2 School of Food and Wine, Shenyang Pharmaceutical University, 103 Wenhua Road, Shenyang 110016, China; \\ yintian124@vip.163.com \\ * Correspondence: hehaibing@syphu.edu.cn
}

Citation: Song, Z.; Yin, J.; Xiao, P.; Chen, J.; Gou, J.; Wang, Y.; Zhang, Y.; Yin, T.; Tang, X.; He, H. Improving Breviscapine Oral Bioavailability by Preparing Nanosuspensions, Liposomes and Phospholipid Complexes. Pharmaceutics 2021, 13, 132. https://doi.org/10.3390/ pharmaceutics 13020132

Received: 21 December 2020

Accepted: 16 January 2021

Published: 20 January 2021

Publisher's Note: MDPI stays neutral with regard to jurisdictional claims in published maps and institutional affiliations.

Copyright: (c) 2021 by the authors. Licensee MDPI, Basel, Switzerland. This article is an open access article distributed under the terms and conditions of the Creative Commons Attribution (CC BY) license (https:// creativecommons.org/licenses/by/ $4.0 /)$.

\begin{abstract}
Breviscapine (BVP), a flavonoid compound, is widely used in the treatment of cardiovascular and cerebrovascular diseases; however, the low oral bioavailability and short half-life properties limit its application. The aim of this study was to investigate the three preparations for improving its oral bioavailability: nanosuspensions (BVP-NS), liposomes (BVP-LP) and phospholipid complexes (BVP-PLC). In vitro and in vivo results suggested that these three could all significantly improved the cumulative released amount and oral bioavailability compared with physical mixture, in which BVP-PLC was the most optimal preparation with the relative bioavailability and mean retention time of $10.79 \pm 0.25(p<0.01)$ and $471.32 \%(p<0.01)$, respectively. Furthermore, the influence of drug-lipid ratios on the in vitro release and pharmacokinetic behavior of BVP-PLC was also studied and the results showed that 1:2 drug-lipid ratio was the most satisfactory one attributed to the moderateintensity interaction between drug and phospholipid which could balance the drug loading and drug release very well.
\end{abstract}

Keywords: breviscapine; oral bioavailability; nanosuspensions; liposomes; phospholipid complexes

\section{Introduction}

Breviscapine (BVP) is a flavonoid compound extracted from the Chinese herb Erigeron breviscapus (Vant) Hand-Mazz. Scutellarin (>85.0\%), 4,5,6-tetrahydroxyflavone-7-O-glucuronide (Figure 1), is a flavonoid glycoside compound and is the main active ingredient of BVP [1]. BVP has various pharmacological activities, including dilating blood vessels, increasing cardiovascular and cerebrovascular blood flow, anti-platelet aggregation and improving microcirculation [2,3], anti-inflammatory [4], renoprotective and neuroprotective effects $[1,5-8]$. Therefore, BVP products are widely used in the treatment of cardiovascular and cerebrovascular diseases such as coronary artery disease, myocardial infarction, hypertension, hyperlipidemia and diabetic complications in China [9]. Cerebrovascular and cardiovascular diseases are chronic diseases which require drugs with good bioavailability and long half-life. Unfortunately, BVP belongs to the Biopharmaceutical Classification System (BCS) class IV drugs and its half-life is very short [10,11]. Studies have shown that there are several main causes of the low bioavailability of BVP: (1) low oral solubility, limited membrane permeability, transporter mediated drug efflux [12] and first-pass elimination in gastrointestinal tract (GIT) [13,14]; (2) the hydroxyl groups in 5, 6, 7 and $4^{\prime}$ positions of BVP can combine with endogenous- $\alpha$-D-glucuronic acid to form glucuronic glycosides which results rapidly metabolized [15]; (3) metabolic enzymes and $\mathrm{pH}$-mediated environmental degradation in GIT; (4) wide distribution in body and diversity of biological transformation $[16,17]$. In recent years, researchers have prepared various formulations to 
improve the oral bioavailability of BVP by using nanoemulsions, nanoparticles, liposome nanocomposite particles, phospholipid complexes and more [18-20].<smiles>O=C(O)[C@H]1OC(Oc2cc3oc(-c4ccc(O)cc4)cc(=O)c3c(O)c2O)[C@H](O)[C@@H](O)[C@H]1O</smiles>

Figure 1. The structure of scutellarin.

Nanotechnology is an effective tool for improving the bioavailability and bioactivity of many water-insoluble drugs, for example flavonoids, as it can endow the preparations with reduced particle sizes [21]. Nanosuspensions are defined as a carrier-free drug delivery system usually consisting of a stabilizer and active pharmaceutical ingredient (API), which possesses high drug loading capacity and satisfactory biocompatibility [22]. Liposomes are a kind of spherical vesicles which have biofilm-like bilayer consisting of phospholipids and cholesterols and so forth. They have a size range from $\sim 20 \mathrm{~nm}$ to $\sim 5 \mu \mathrm{m}$ in diameter, with one or more lipid bilayers surrounding aqueous compartments [23]. Liposomes have several desirable properties, such as good biodegradability and biocompatibility, prolonged release effect and enhanced chemical stability of drugs [24-31]. Phospholipid complexes, also named phytosomes, are a novel drug delivery system formed by non-covalent bonds (electrostatic interaction, van der Waals force, hydrogen bond and etc.) between phospholipids and drugs [32].The structure of phospholipid complexes is similar to that of liposomes, with the major difference being that in phospholipid complexes, the drug is mainly connected with the phospholipid polar head, whereas in liposomes, the drug is encapsulated in its cavity or phospholipid bilayer [33]. Drug-phospholipid complexes possess amphiphilic characteristics, which makes them more readily soluble in GIT and more easily absorbed through amphiphilic cell membrane and lymphatic system, thereby improving the bioavailability of drugs. Furthermore, phospholipid complexes can increase drug stability, extend the action duration of drugs and delay drug elimination [32]. Last several years, phospholipid complexes have been widely used to improve oral bioavailability of poorly water-soluble drugs, for example quercetin [34], kaempferol [35], silybin [36], curcumin [37] and tamoxifen [38].

In this study, three preparations, breviscapine nanosuspensions (BVP-NS), breviscapine liposomes (BVP-LP) and breviscapine phospholipid complexes (BVP-PLC) were prepared for oral administration to increase the drug absorption and extend the half-life in order to improve bioavailability. The particle size and zeta potential were measured by dynamic light scattering (DLS), besides the morphology was observed by use of transmission electron microscopy (TEM), the results indicated that the particle sizes were about 200-300 nm and zeta potentials were all negative. The existence form of BVP in different preparations was investigated by polarized light microscopy (PLM), differential scanning calorimetry (DSC) and powder X-ray diffraction (PXRD), which suggested that BVP was existed in crystal form in NS, while as non-crystal form in LP and PLC. An FT-IR experiment was carried out to investigated the interactions between BVP and phospholipid molecule, the results demonstrated there really existed interactions. Molecule simulation further indicated the $-\mathrm{OH}$ and $-\mathrm{COO}^{-}$group in BVP could form hydrogen bond and electrostatic interaction with phospholipid molecule in PLC, rather than NS and LP. In vitro release results manifested that BVP-PLC showed the highest cumulative release amount of, followed by BVP-NS and BVP-LP. In vivo pharmacokinetic study verified that BVP-NS, BVP-LP and BVP-PLC all shown significantly higher oral bioavailability compared with 
BVP. In addition, BVP-LP and BVP-PLC exhibited a distinctly extended-release profile. Furthermore, the drug-lipid ratio influence on the pharmacokinetic parameters of BVPPLC was also investigated, in which the preparation with drug-lipid ratio of 1:2 had the highest relative bioavailability. As the higher drug-lipid ratio (1:1) could not provide strong enough force to maintain the stability of the preparation and the lower drug-lipid ratio (1:3) impacted the dissociation of BVP from the preparation. Therefore, phospholipid complexes with appropriate drug-lipid ratio provide an effective strategy for improving the oral bioavailability of drugs which have low solubility both in water and lipid. The Figure 2 illustrated overall scheme of this work and the mechanism of the three preparations to improve bioavailability.

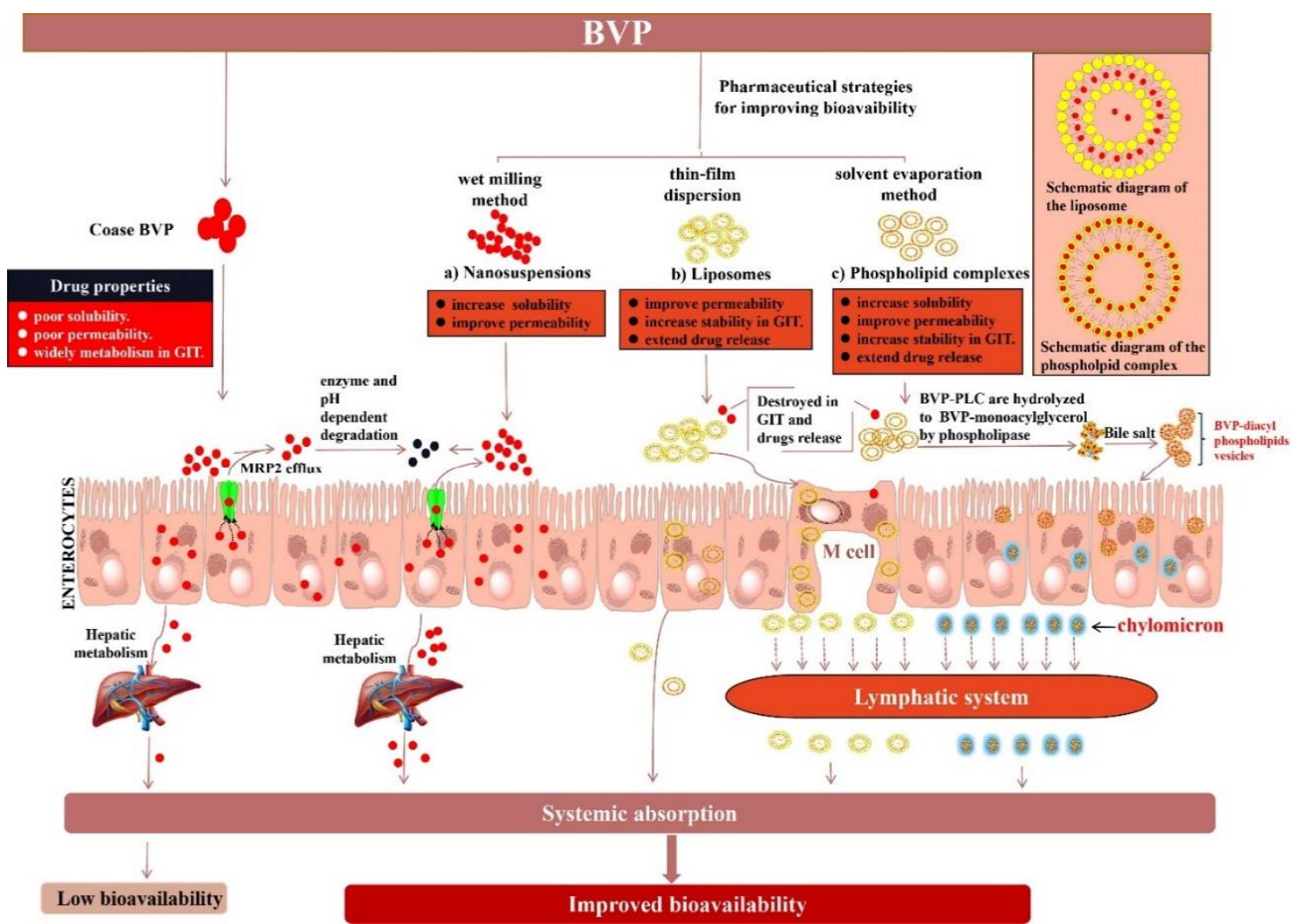

Figure 2. The outline of this paper and the mechanism of Breviscapine-nanosuspension (BVP-NS), BVP-liposomes (LP) and BVP-phospholipid complexes (PLC) to enhance bioavailability.

\section{Materials and Methods}

\subsection{Materials and Animals}

BVP (scutellarin purity $>$ 98.5\%) was purchased from Kunming Longjin Pharmaceutical Co., Ltd. (Yunnan, China). Soybean phospholipid was provided by Shenyang Tianfeng Bio-Pharmaceutical Co., Ltd. (Shenyang, China). Hydroxypropyl methylcellulose E5 (HPMC-E5) was obtained from Dow Chemicals Company (Midland, MI, USA). Scutellarin reference substance and baicalin (internal standard, IS) were provided by National Institute of Food and Drug Control (Beijing, China). Mannitol was supplied by Tianjin Heng Xing Chemical Reagent Co., Ltd. (Tianjin, China). Cholesterol was supplied by Hennan Liwei Biological Pharmaceutical Co., Ltd. (Jiaozuo, China). Vitamin E was provided by Zhejiang NHU Company Co., Ltd. (Xinchang, China). All other chemicals and reagents used were of analytical or chromatographic quality and deionized water was used throughout the study.

Male Sprague-Dawley rats were provided by Liaoning Changsheng Biotechnology Co., Ltd. (Shenyang, China). All the animal research was approved by the Committee of 
Ethics of Animal Experimentation of Shenyang Pharmaceutical University and carried out in accordance with the guidelines of this committee (SYPU-IACUC-C2019-7-3-204).

\subsection{Preparation of BVP-NS, BVP-LP and BVP-PLC}

\subsubsection{BVP-NS}

The BVP-NS were prepared by wet-milling method [39]. BVP (3.0 g) and HPMCE5 $(0.6 \mathrm{~g})$ were weighed and dispersed in a small amount of distilled water. Separately, soybean lecithin $(6.0 \mathrm{~g})$ was dissolved in $7.5 \mathrm{~mL}$ of absolute ethanol and stirring (DF-101S, Gong yi yu hua Instrument Co. LTD, Gongyi , China) at $60{ }^{\circ} \mathrm{C}$, after the solution turn into yellow and clear, the water phase was added in and then the mixture was diluted by distilled water to $150 \mathrm{~mL}$. Then, transferred into a Superfine Grinding Equipment (Minieasy, Retsch Topway Technology Co., Ltd., Beijing, China) with zirconium oxide milling beads $(0.6-0.8 \mathrm{~mm}$ diameter) as the milling media and milling for $2 \mathrm{~h}$ at $2000 \mathrm{rpm}$. The final suspension was spray-dried to obtain a solid dosage form with $2.5 \%$ mannitol as protective agent.

\subsubsection{BVP-LP}

The thin-film dispersion method was used to prepare BVP-LP $[24,25,27,28] .1 .0 \mathrm{~g}$ phospholipid and $0.25 \mathrm{~g}$ cholesterol were accurately weighed and dissolved in absolute ethanol. The organic solvent was evaporated at $50{ }^{\circ} \mathrm{C}$ for $30 \mathrm{~min}$ to form a thin film and then hydrated using $100.0 \mathrm{~mL}$ of phosphate buffer solution (pH 7.4) containing $200.0 \mathrm{mg}$ BVP for $1 \mathrm{~h}$ at $55^{\circ} \mathrm{C}$ until all the lipids were completely dispersed into the aqueous phase. The crude liposome dispersion was subjected to ultrasound for $3 \mathrm{~min}$ (on $2.5 \mathrm{~s}$, off $3 \mathrm{~s}$ ) by a SCIENTZ-IID Ultrasonic Cell Crusher (Xinzhi Biological Technology Co., Ltd., Ningbo, China) at $280 \mathrm{~W}$ in ice bath. After the free BVP was removed as method described in 2.4, the final liposome dispersion obtained. Next, $10 \%(w / v)$ mannitol as the lyoprotectants were added and filtered through a $0.22 \mu \mathrm{m}$ filter to remove insoluble impurities. The filtered preparations were lyophilized at $-20^{\circ} \mathrm{C}$ for $24 \mathrm{~h}$ to obtain the freeze-dried BVP-LP powder. The blank liposome was prepared by the same method except that BVP was not added.

\subsubsection{BVP-PLC}

BVP-PLC was prepared by a solvent evaporation method [32]. Briefly, BVP $(0.60 \mathrm{~g})$ and appropriate phospholipid were weighed at different mass ratios (1:1, 1:2, 1:3) and dissolved in $150 \mathrm{~mL}$ absolute ethanol in a $250 \mathrm{~mL}$ round bottom flask, with appropriate amount of Vitamin E added in as antioxidant. The mixture was refluxed at $50{ }^{\circ} \mathrm{C}$ for $2 \mathrm{~h}$. Subsequently, the resultant clear solution was completely evaporated by spray drying with $2.5 \%$ mannitol as protective agent.

\subsection{Encapsulation Efficiency (EE) of BVP-LP}

The liposomes and free BVP were isolated by ultrafiltration. $4.0 \mathrm{~mL}$ preparation was accurately measured and placed in an ultrafiltration tube with cutoff molecular weight of $100 \mathrm{kDa}$ and centrifuged at $3000 \mathrm{rpm}$ for $20 \mathrm{~min}$. The encapsulated BVP-LP remained on the surface of the filter membrane and free drug was collected into the bottom of the filter [40]. The separated bottom phases were transferred to $10 \mathrm{~mL}$ volumetric flask and diluted to the volume with methanol. After filtration by a $0.22 \mu \mathrm{m}$ microporous filtration membrane, the samples and reference solution were qualified by HPLC (Agilent $1260 \mathrm{~L}, \mathrm{C} 18$ column (Hypersil GOLD C18, $250 \times 4.6 \mathrm{~mm}^{2}, 5 \mu \mathrm{m}$, Thermo, USA), UV detector (set at $335 \mathrm{~nm}$, Agilent Technologies Inc., California, USA) and the mobile phase was methanol/0.1\% phosphoric acid solution $(40: 60, v / v)$ with a flow rate of $1.0 \mathrm{~mL} / \mathrm{min}$. The EE of BVP-LP was determined by Equation (1).

$$
E E=\frac{\text { The mass of total BVP }- \text { the mass of free BVP }}{\text { The mass of total BVP }} \times 100 \%
$$




\subsection{Complexation Efficiency (CE) of BVP-PLC}

The CE of phospholipid complexes is an important parameter. Due to the different solubilities in chloroform, the CE of phospholipid complexes could be measured, in which free BVP is insoluble but phospholipid complexes are readily soluble [36]. The phospholipid complexes (equivalent to $10.0 \mathrm{mg}$ of BVP) was accurately weighed and then dissolved in $10.0 \mathrm{~mL}$ chloroform and sonicated for $30 \mathrm{~min}$. After centrifugation at $3000 \mathrm{rpm}$ for $5 \mathrm{~min}$, the supernatant was collected and filtered using a $0.22 \mu \mathrm{m}$ filter. $1.0 \mathrm{~mL}$ of the filtrate was accurately measured and transferred into a $50 \mathrm{~mL}$ round bottom flask. After the chloroform removed by rotary evaporation, appropriate amount of methanol was added to dissolve the precipitation. Next, the mixture was transferred into a $10 \mathrm{~mL}$ volumetric flask and diluted to the volume with methanol. The amount of BVP encapsulated was determined by HPLC as above mentioned. In order to obtain the total amount of BVP, the phospholipid complexes (equivalent to $10.0 \mathrm{mg}$ of BVP) was dissolved by appropriate amount of methanol and transferred into a $10 \mathrm{~mL}$ volumetric flask and diluted to the volume with methanol and determined as above described. The complexation efficiency (CE) was defined by Equation (2).

$$
C E=\frac{\text { The amount } f \text { combined } B V P}{\text { The amount of total } B V P} \times 100 \%
$$

\subsection{Characterization}

2.5.1. Determination of Particle Size and Zeta Potential

Mean particle size, polydispersity index (PDI) and zeta potential of BVP-NS, BVPLP and BVP-PLC were measured using Zeta sizer nano-ZS 900 (Malvern Instruments, Malvern, UK) (medium:water; dispersant refractive index(RI): 1.33cP; Viscosity: 0.8872; dielectric constant: $78.36 \mathrm{~F} / \mathrm{m}$; count rate: 200-400; material RI: 1.59; wavelength: 633 $\mathrm{nm})$. BVP-NS, BVP-LP or BVP-PLC were dispersed in purified water and diluted to an appropriate concentration. Each sample was measured in triplicate at room temperature and the result was expressed as mean $\pm \mathrm{SD}$.

\subsubsection{Polarized Light Microscopy (PLM) Analysis}

PLM was used to characterize the BVP-NS, BVP-LP and BVP-PLC (1:2). The mannitol, HPMC-E5, soybean lecithin, cholesterol, BVP, BVP-NS, BVP-LP, BVP-PLC (1:2) and three physical mixtures (the first (PM-BVP-NS) consisting of BVP, soybean lecithin, HPMC-E5 and mannitol; the second (PM-BVP-LP) consisting of BVP and blank liposome; the third (PM-BVP-PLC (1:2)) consisting of BVP, phospholipid and mannitol) were observed using positive polarizing microscope (Leica DM2700P, Leica Microsystems, Wetzlar, Germany) at a $90^{\circ}$ angle between the lifting mirror and the detecting mirror (vertical).

\subsubsection{Transmission Electron Microscopy (TEM) Analysis}

BVP-NS, BVP-LP and BVP-PLC (1:2) were negatively stained with $2 \%$ phosphotungstic acid and placed on a copper grid coated with carbon film and then the morphologies were observed using TEM (Hitachi HT7700, Hitachi, Ltd., Tokyo, Japan).

\subsubsection{Fourier Transform Infrared Spectroscopy (FT-IR) Analysis}

The samples of mannitol, HPMC-E5, soybean lecithin, cholesterol, BVP, PM-BVP-NS, BVP-NS, PM-BVP-LP, BVP-LP, PM-BVP-PLC (1:2) and BVP-PLC (1:2) were prepared by $\mathrm{KBr}$ pressing method for the measurement by Fourier transform infrared (FTIR) spectrophotometer (EQUINOX 55, Bruker, Karlsruhe, Germany).

\subsubsection{Differential Scanning Calorimetry (DSC) Analysis}

The thermal behavior of mannitol, HPMC-E5, soybean lecithin, cholesterol, BVP, PM-BVP-NS, BVP-NS, PM-BVP-LP, BVP-LP, PM-BVP-PLC (1:2) and BVP-PLC (1:2) were analyzed using DSC-3 (METTLER TOLEDO, Zurich, Switzerland). The samples were 
sealed in crimped standard aluminum pans and heated under nitrogen flow (50 $\mathrm{mL} / \mathrm{min})$ at a rate of $10{ }^{\circ} \mathrm{C} / \mathrm{min}$ from $30^{\circ} \mathrm{C}$ to $300{ }^{\circ} \mathrm{C}$.

\subsubsection{Powder X-ray Diffraction (PXRD) Analysis}

The polymorphic state of mannitol, HPMC-E5, soybean lecithin, cholesterol, BVP, PM-BVP-NS, BVP-NS, PM-BVP-LP, BVP-LP, PM-BVP-PLC (1:2) and BVP-PLC (1:2) were determined by a D/Max-2400 X-ray diffractometer (Rigaku, Osaka, Japan). A continuous scan mode was run with a rate of $2^{\circ} /$ min over a $2 \theta$ range from $5^{\circ}$ to $60^{\circ}$ and data were collected with $\mathrm{Cu}-\mathrm{Ka}$ radiation and a voltage of $56 \mathrm{kV}$.

\subsection{In Vitro Release Studies}

The in vitro release of BVP-NS, BVP-LP and BVP-PLC were carried out by ZRS-8G dissolution apparatus (Tianjin Tianda Tianfa Technology Co., Ltd, Tianjin, China). The dissolution medium was $\mathrm{pH} 6.8$ phosphate buffer solution, the rotation speed was $50 \mathrm{rpm}$ and the temperature was $37 \pm 0.5^{\circ} \mathrm{C}$. Predetermined amounts of BVP-NS, BVP-LP or BVP-PLC (drug mass approximately $36 \mathrm{mg}$ ) were added in $900 \mathrm{~mL}$ dissolution medium. $5 \mathrm{~mL}$ release medium was sampled at the time point of 5, 15, 30, 45, 60, 90 and $120 \mathrm{~min}$ and $5 \mathrm{~mL}$ of fresh dissolution medium was added to maintain sink condition. The content was determined by HPLC as previous described and the cumulative dissolution percentage of the drug at each time point was calculated using the external standard method.

\subsection{Pharmacokinetic Studies}

In order to compare the oral bioavailability of BVP in the three formulation strategies, the pharmacokinetic study was performed. Male Sprague-Dawley rats weighing from 200 to $220 \mathrm{~g}$ were randomly divided into four groups $(n=4)$. Each drug solution in the different formulations (the formulations were dispersed with $0.5 \%$ sodium carboxymethyl cellulose) was administered to rats by gavage (20 mg/kg BVP): Group A (BVP), Group B (BVP-PLC), Group C (BVP-NS) and Group D (BVP-LP (1:2)). Prior to the experiment, the SD rats were fasted for $12 \mathrm{~h}$ but had free access to water. After gavage administration, $0.5 \mathrm{~mL}$ blood were sampled at $0.25,0.5,1,2,4,6,8,10,12,24 \mathrm{~h}$ and transferred into a heparinized centrifuge tube. The collected samples were centrifuged at $5000 \mathrm{rpm}$ for $10 \mathrm{~min}$ at $4{ }^{\circ} \mathrm{C}$ and the supernatant plasma was analyzed by UPLC-MS/MS (Waters Corp, Manchester, UK). Food was provided to the rats $4 \mathrm{~h}$ after of administration. At the same time, a pharmacokinetic study was also performed to compare the oral bioavailability of BVP-PLC with different drug-lipid ratios (1:1, 1:2, 1:3).

The plasma concentration of BVP was measured using ultrahigh performance liquid chromatography tandem mass spectrometry (UPLC-MS/MS) [41]. An ACQUITY UPLC system (Waters Corp., Milford, MA, USA) and Thermo C18 column $(50 \mathrm{~mm} \times 2.1 \mathrm{~mm}$ ID, $1.7 \mu \mathrm{m}$, Thermo Fisher SCIENTIFIC, Thermo, USA) was used for the chromatographic separations, with a gradient elution of methanol and $0.1 \%$ formic acid solution (Table 1 ). Mass spectrometric determination was performed in positive ESI mode and the compounds were quantified by multiple reaction monitoring (MRM, Waters Corp, Manchester, UK) of the transitions of $\mathrm{m} / \mathrm{z} 463 \rightarrow 287.1$ for BVP and $\mathrm{m} / \mathrm{z} 447.1 \rightarrow 271.1$ for Baicalin as IS, respectively.

Table 1. UPLC-MS/MS gradient elution method for BVP analysis.

\begin{tabular}{cccc}
\hline Time (min) & Flow Rate $(\mathbf{m L} / \mathbf{m i n})$ & $\mathbf{\% A}$ & $\mathbf{\%} \mathbf{B}$ \\
\hline 0.0 & 0.2 & 60 & 40 \\
0.5 & 0.2 & 60 & 40 \\
1.2 & 0.2 & 25 & 75 \\
1.8 & 0.2 & 5 & 95 \\
2.8 & 0.2 & 5 & 95 \\
3.0 & 0.2 & 60 & 40 \\
3.5 & 0.2 & 60 & 40 \\
\hline
\end{tabular}




\subsection{Statistical Analysis}

All data are expressed as the mean and standard deviation (mean \pm SD). The statistical significance of the differences between the groups was assessed by non-parametric statistics-Kruskal-Wallis ANOVA with post-hoc test. Analysis of variance was used for comparing more than two samples, where $p<0.05$ and $p<0.01$ (two-tailed) were considered statistically significant and extremely significant, respectively. Pharmacokinetic parameters were obtained with a non-compartmental method using drug and statistics (DAS) software.

\section{Results and Discussion}

\subsection{Characterization}

\subsubsection{Particle Size and Zeta Potential}

The reduction of drug particle size into nanoscale is an effective method to enhance oral bioavailability. However, with the decrease of particle size, the increase of surface energy would lead to particle aggregation and deposition. The addition of stabilizers and an appropriate zeta potential are beneficial to the stability of the preparation. The particle size, PDI and zeta potential of three preparations were summarized in Table 2. The particle size of all three preparations was about $300 \mathrm{~nm}[42,43]$ and the PDI of three preparations were all smaller than 0.3. The zeta potentials were all negative, which could provide effective prevention of aggregation of particles to maintain a homogenous preparation. It was worthy to note that the particle size of BVP-PLC tended to increase with the decrease of the drug-lipid ratio, which may be caused by the excessive phospholipids attached to the phospholipid complexes.

Table 2. Mean particle size, $\mathrm{PDI}, \mathrm{CE} \%$, EE\% and zeta potential/mV of three preparations.

\begin{tabular}{cccccc}
\hline Formulation & $\begin{array}{c}\text { Drug-Lipid } \\
\text { Radio }\end{array}$ & $\begin{array}{c}\text { Mean Particle } \\
\text { Size/nm }\end{array}$ & PDI & EE\%/CE\% & $\begin{array}{c}\text { Zeta } \\
\text { Potential/mV }\end{array}$ \\
\hline BVP-NS & - & $277.4 \pm 5.6$ & $0.234 \pm 0.070$ & - & $-15.2 \pm 0.6$ \\
BVP-LP & - & $265.3 \pm 13.0$ & $0.221 \pm 0.058$ & $76.41 \pm 5.61$ & $-13.1 \pm 1.3$ \\
BVP-PLC & $1: 1$ & $272.2 \pm 20.1$ & $0.29 \pm 0.068$ & $61.31 \pm 2.32$ & $-20.5 \pm 0.8$ \\
BVP-PLC & $1: 2$ & $298.6 \pm 36.6$ & $0.278 \pm 0.051$ & $72.90 \pm 3.21$ & $-22.2 \pm 1.0$ \\
BVP-PLC & $1: 3$ & $338.4 \pm 26.7$ & $0.266 \pm 0.047$ & $79.36 \pm 3.76$ & $-21.0 \pm 0.9$ \\
\hline
\end{tabular}

\subsubsection{EE (\%) of BVP-LP and CE (\%) of BVP-PLC}

Since BVP is a weak acidic drug, unencapsulated BVP could dissolved in weak alkaline solution and therefore the $\mathrm{EE}$ was determined by ultrafiltration centrifugation method. The EE of BVP-LP with SPC/Chol weight ratio of $4: 1$ was $76.41 \pm 5.61 \%$ (Table 2). The CE represents an extremely important index for prescription screening of BVP-PLC. The determination of the $\mathrm{CE}$ is based on different solubility of formed phospholipid complexes and the free drug in chloroform, in which the phospholipid complexes could readily dissolve while the free BVP remains highly insoluble [44]. As shown in Table 2, the CE of BVP-PLC increased as the drug-lipid ratio decreasing, with the highest of $79.36 \pm 3.76 \%$ at drug-lipid ratio of 1:3.

\subsubsection{TEM}

The morphologies of BVP-NS, BVP-LP and BVP-PLC (1:2) observed by transmission electron microscopy (TEM) were shown in Figure 3. The results indicated that all samples exhibited a spherical shape. It is well known that when phospholipids are dispersed in an aqueous solution, they can self-assemble to form ordered structures such as micelles, vesicles, liposomes, bilayer membranes and planar phospholipid multilayer membranes according to the different environments (for example, different concentrations of phospholipids). For BVP-NS, a large amount of soybean phospholipid was added as stabilizer. The majority of drugs was encapsulated by phospholipids and could form spherical particles after wet grinding (Figure 3a,b). For BVP-LP, the morphology a little differed from BVP-NS 
and BVP-PLC (1:2), which presented a fingerprint-like internal structure with a size around $200 \mathrm{~nm}$ (Figure 3c,d). The TEM images revealed the morphology of the BVP-PLC (1:2) was in globular shape with no internal structures, suggesting that the actual structure of BVP-PLC may be vesicles or micelles (Figure 3e,f). Anisha Mazumder et al. also confirmed that sinigrin-phytosome complexes formed vesicle-like structures which were ellipsoidal or spherical, self-closed, well-identified, well-formed and dispersed by TEM [45].
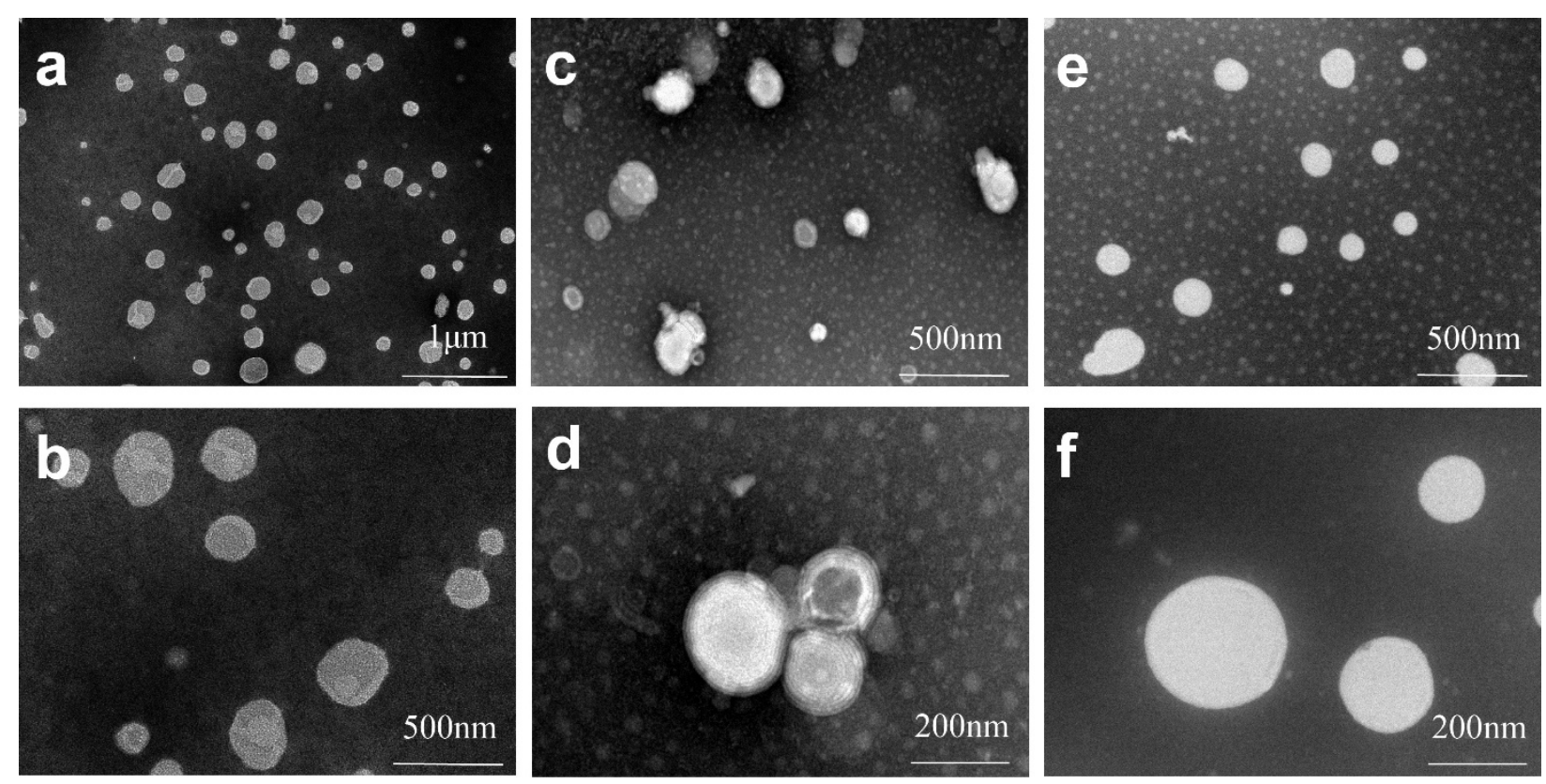

Figure 3. Transmission electron microscopy (TEM) images of BVP-NS (a,b), BVP-LP(c,d) and BVP-PLC (1:2) (e,f).

\subsubsection{PLM Analysis}

Polarized light microscopy (PLM) was often used to evaluate the birefringence phenomenon of crystal drugs, amorphous drugs and polymer materials $[43,46]$. If the matters were amorphous, there will be no birefringence and the field of vision will be dark. PLM results of ingredients and different formulations were shown in Figure 4. The ingredients of mannitol, HPMC-E5, cholesterol and BVP showed significant birefringence phenomenon except for soybean lecithin which is amorphous and therefor the field is dark. For BVPNS, the birefringence phenomenon of BVP still existed but a little weak compared with PM-BVP-NS, indicated that most BVP was still in crystal form but part of the drug was transformed into an amorphous form during nano-milling and/or the spray drying process [46]. The result of PM-BVP-LP (1:2) showed distinct birefringence phenomenon of mannitol, cholesterol and BVP, while in BVP-LP, the BVP signal weakened or even disappeared, indicating that the drug was not exist in the form of crystal in liposomes. According to the property of BVP and structure of liposome, the drug was very likely to exist in the form of molecules between the lipid bilayer or in the internal aqueous phase. The result of BVP-PLC (1:2) almost had no BVP signal compared with PM-BVP-PLC (1:2), indicating that the drug in BVP-PLC (1:2) was also not in crystal form, which may present in a highly dispersed molecular state. 


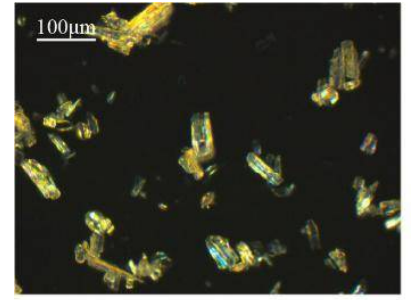

Mannitol

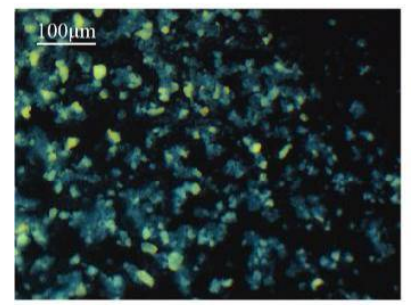

BVP

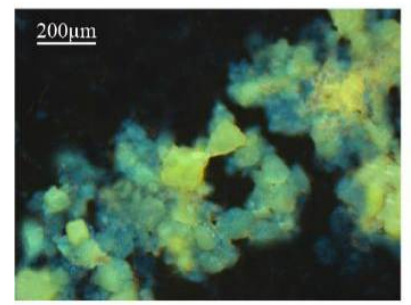

BVP-LP

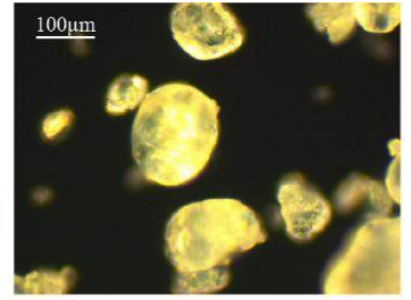

HPMC-E5

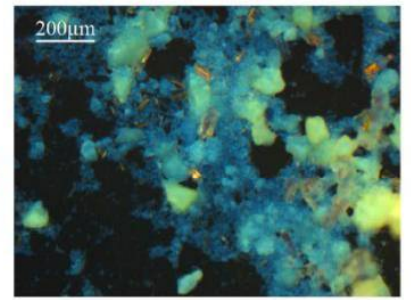

PM-BVP-NS

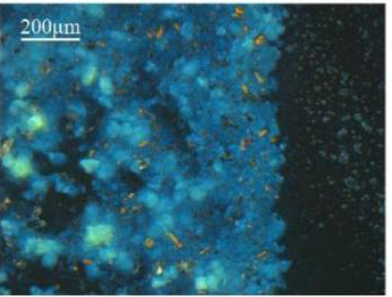

PM-BVP-PLC(1:2)

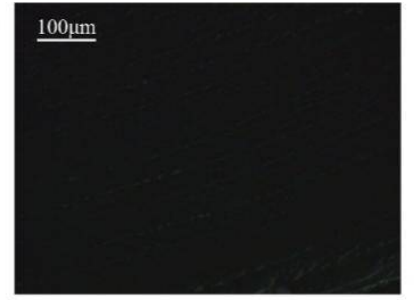

Soybean lecithin

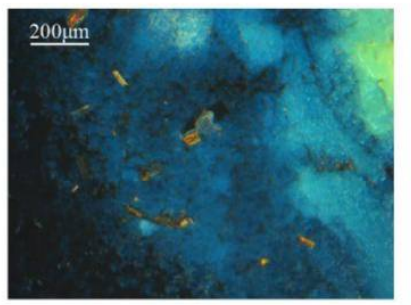

BVP-NS

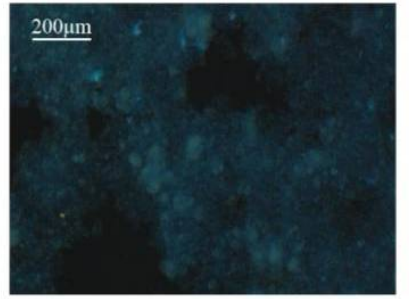

BVP-PLC(1:2)

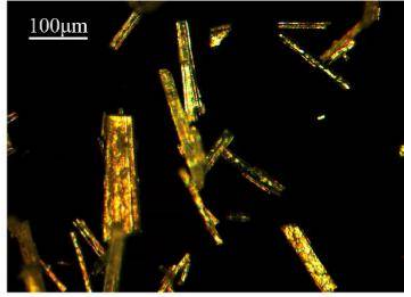

Cholesterol

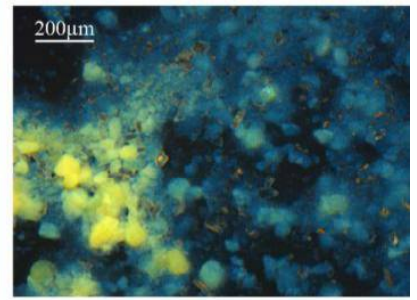

PM-BVP-LP

Figure 4. Polarized light microscopy (PLM) images of mannitol, HPMC-E5, soybean lecithin, cholesterol, BVP, PM-BVP-NS, BVP-NS, PM-BVP-LP, BVP-LP, PM-BVP-PLC (1:2) and BVP-PLC (1:2).

\subsubsection{FT-IR Analysis}

Fourier transform infrared spectroscopy (FT-IR) is an effective method to characterize molecular structure and is widely used to study molecular interactions between drugs and excipients. As shown in Figure 5, the BVP exhibited characteristic FT-IR absorption peaks at $3509.6,3373.8$ and $3275.5 \mathrm{~cm}^{-1}$ (-OH stretching vibration), $1720.9 \mathrm{~cm}^{-1}$ (C=O stretching vibration), $1661.3 \mathrm{~cm}^{-1}$ and $1600 \mathrm{~cm}^{-1}$ (C=C stretching vibration), $1360.4 \mathrm{~cm}^{-1}$ and $1083.0 \mathrm{~cm}^{-1}$ (C-O deformation vibration) and $1222.8 \mathrm{~cm}^{-1}$ (C-O-H stretching vibration). For BVP-NS and BVP-LP, all characteristic BVP peaks were presented in spectra compared with PM-BVP-NS and PM-BVP-LP, indicating that BVP in the BVP-NS or BVP-LP maintains the original drug structure and there was no detectable interaction.

As for BVP-PLC (1:2), the FT-IR spectra showed a bimodal infrared absorption at 3395.7 and $3286.1 \mathrm{~cm}^{-1}$, which were absorption peaks of hydroxyl and carboxyl groups in the range of $3650-3200 \mathrm{~cm}^{-1}$ and was superimposable with pure BVP and excipients. However, compared with BVP and PM-BVP-PLC (1:2), the BVP-PLC (1:2) had one strong wider absorption peak in the range of $3650-3200 \mathrm{~cm}^{-1}$ and meanwhile shifting to lower wave number, suggesting that the hydroxyl group in BVP could form interactions with phospholipid molecules during complexes formation [33]. These findings were in agreement with diosmin lyophilized nano-phytosome [47] described in the literature, where weak interaction between diosmin and phospholipid formed during the preparation of the complexes. 


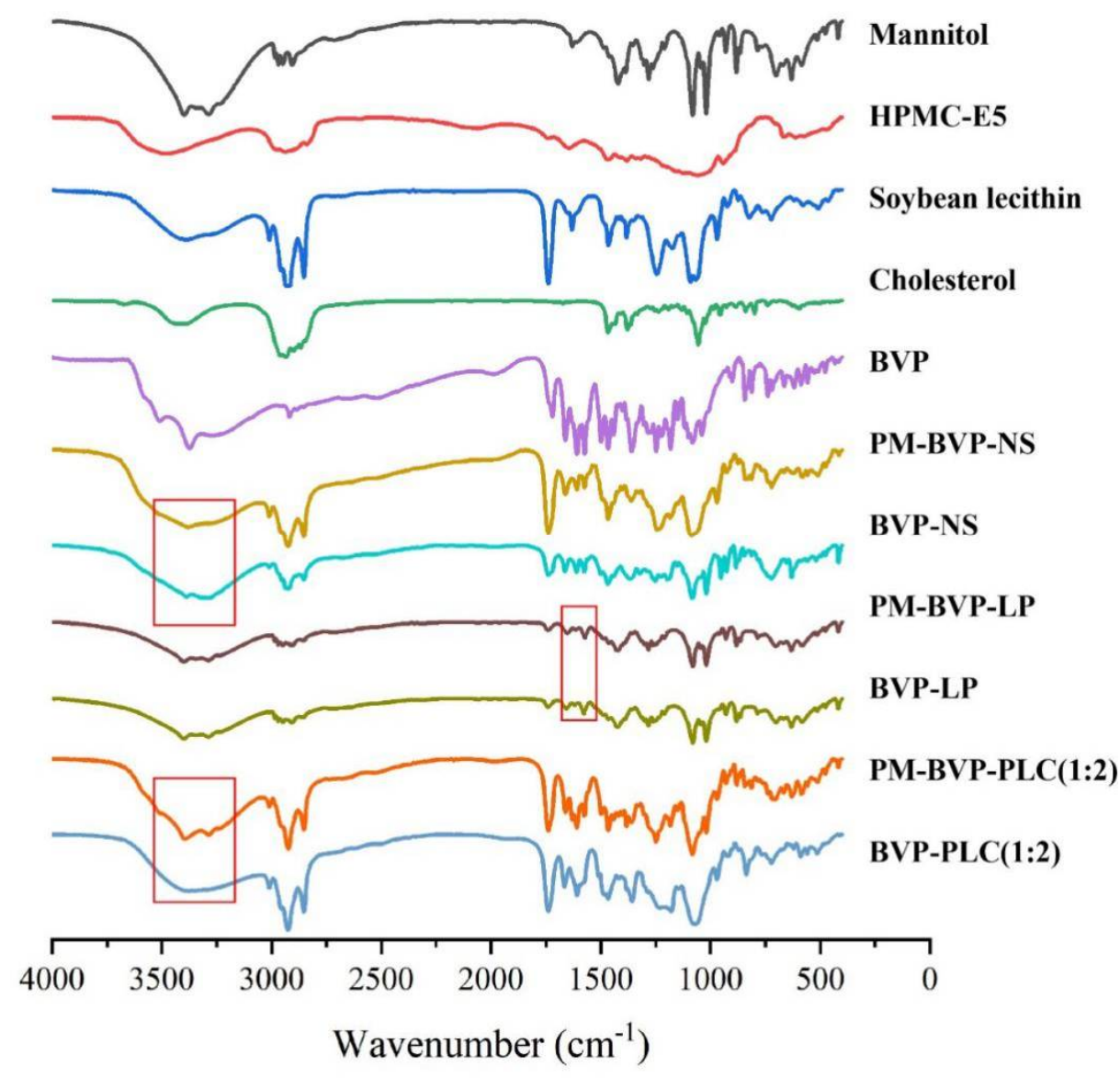

Figure 5. Fourier transform infrared (FTIR) spectrum of mannitol, HPMC-E5, soybean lecithin, cholesterol, BVP, PM-BVP-NS, BVP-NS, PM-BVP-LP, BVP-LP, PM-BVP-PLC (1:2) and BVP-PLC (1:2).

Furthermore, from the perspective of molecular simulation, we could apparently observe the interaction between carboxyl and hydroxyl groups in BVP and phospholipid molecules. As the most representative substance in soybean lecithin, phosphatidyl cholines ( $\mathrm{PC},>60 \%$ ) was used as molecular simulation model to study the interactions between drug and phospholipid in BVP-PLC with different drug-lipid ratios. As shown in Figure 6, the interaction forces between drug and phospholipid were mainly two types, hydrogen bond ( $-\mathrm{OH}$ group in drug and $-\mathrm{P}=\mathrm{O}$ group in phospholipid) and electrostatic interaction $\left(-\mathrm{COO}^{-}\right.$group in drug and $-\mathrm{N}^{+}$in phospholipid) $[32,33]$. In addition to strength, the interaction forms also varied with the drug-lipid ratio. For BVP-PLC (1:1), there were about two molecules of BVP interact with phospholipid, the compact forces were relatively weak. When the drug-lipid ratio raised to 1:2, the molar ratio of phospholipid to drug was about 1:1, one phospholipid molecule compressed one molecule BVP, interaction was strengthened. As for drug-lipid ratio of 1:3 or even lower, the phospholipid in preparation was so overwhelmed that the encapsulation forces were further enhanced, for about two or even more phospholipid molecules to compress one BVP molecule.

\subsubsection{DSC Analysis}

The differential scanning calorimetry (DSC) was also used to determine the exist form of BVP in different preparations. As shown in Figure 7, the thermograms of mannitol and cholesterol exhibited a sharp endothermic peak at $166.1^{\circ} \mathrm{C}$ and $150.3^{\circ} \mathrm{C}$, respectively. BVP showed an endothermic peak at $182.1{ }^{\circ} \mathrm{C}$ and an exothermic peak at $210.3^{\circ} \mathrm{C}$, while HPMC-E5 and soybean lecithin had no obvious endothermic and exothermic peak. 


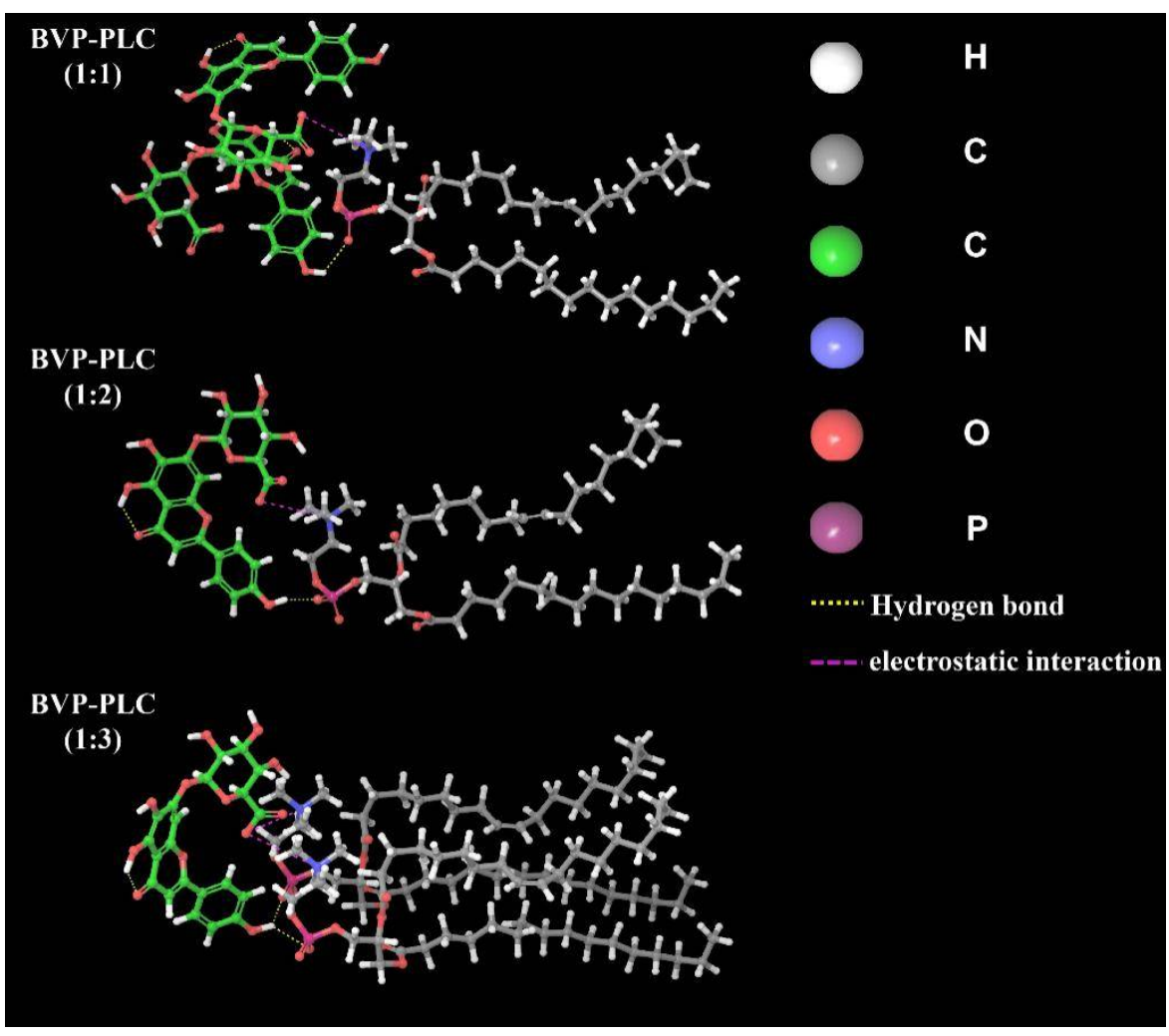

Figure 6. Interaction between drugs and phospholipids in BVP-PLC with different drug-lipid ratios.

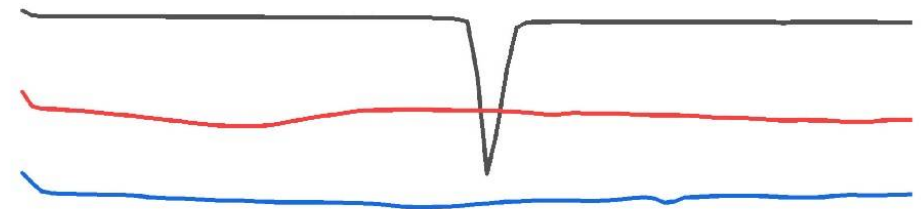

Mannitol

HPMC-E5

Soybean lecithin

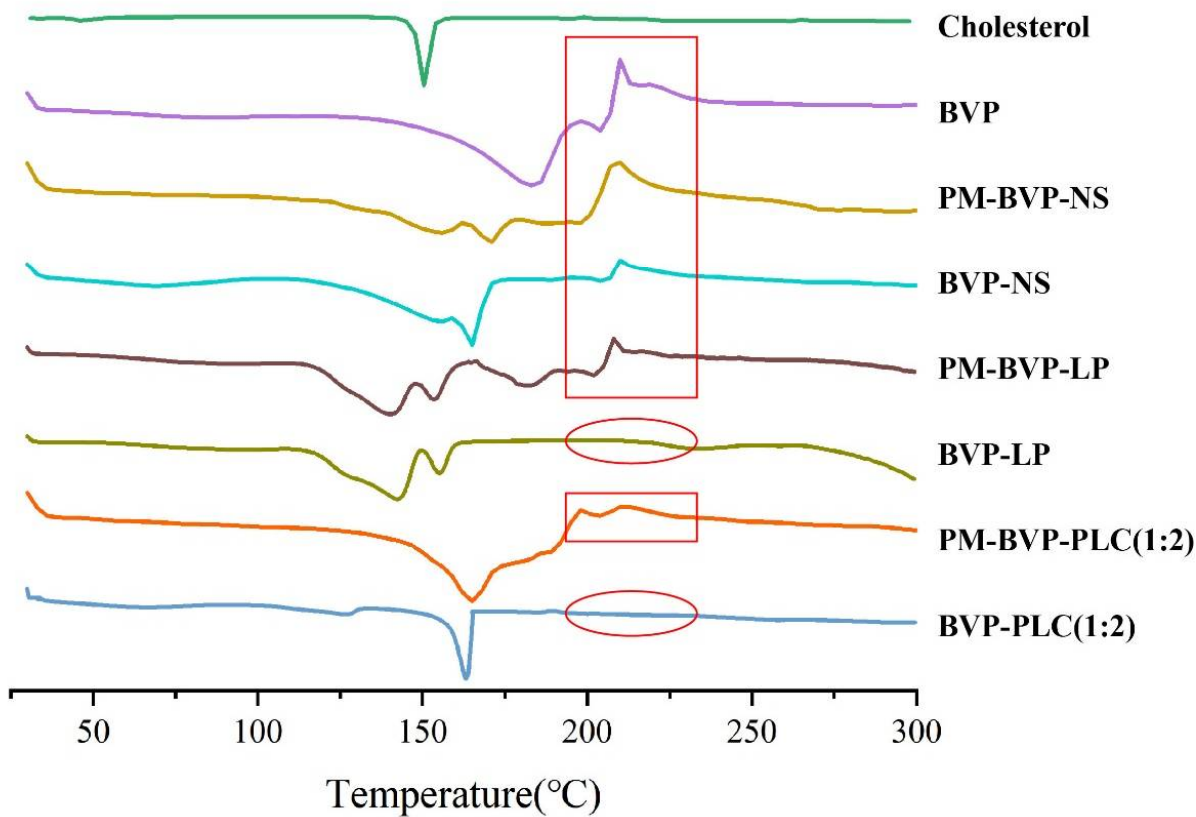

Figure 7. Differential scanning calorimetry (DSC) thermograms of mannitol, HPMC-E5, soybean lecithin, cholesterol, BVP, PM-BVP-NS, BVP-NS, PM-BVP-LP, BVP-LP, PM-BVP-PLC (1:2) and BVPPLC (1:2). 
The DSC curve of BVP-NS was slightly changed compared with BVP and PM-BVP-NS. The characteristic exothermic melting peaks of BVP were still present and the endothermic peaks of BVP was superimposed with mannitol to form a blunt peak in PM-BVP-NS and BVP-NS. The biggest difference for BVP-NS was that the enthalpy at $210.3^{\circ} \mathrm{C}$ was decreased compared with PM-BVP-NS, which indicated that some BVP was transformed into an amorphous state during the milling-drying process [22] and this result can also correspond to PLM. Compared with BVP and PM-BVP-LP, the characteristic peaks of BVP disappeared in the DSC curve of BVP-LP indicated that the BVP existed in non-crystal form. Of note, the peaks of cholesterol and mannitol shifted to $142.3^{\circ} \mathrm{C}$ and $155.2{ }^{\circ} \mathrm{C}$ because of lyophilization, respectively.

While for the BVP-PLC (1:2), the DSC curve of BVP-PLC (1:2) was greatly changed compared with BVP and PM-BVP-PLC (1:2). In the PM-BVP-PLC (1:2) spectrum, the peaks were the simple overlap of BVP, mannitol and soybean lecithin. Whereas in BVP-PLC (1:2), the characteristic absorption melting peaks of BVP at $182^{\circ} \mathrm{C}$ and $210^{\circ} \mathrm{C}$ disappeared and instead showed a sharp absorption peak at $165.34^{\circ} \mathrm{C}$ (mannitol characteristic peak), indicating that BVP-PLC (1:2) formed by BVP and phospholipids was not a simple physical mixture but a new binding state.

\subsubsection{PXRD Analysis}

To further confirm the crystalline state of BVP in BVP-NS, BVP-LP and BVP-PLC (1:2), PXRD analysis was conducted and the X-ray diffractograms were shown in Figure 8. For BVP, characteristic sharp peaks were seen at $2 \theta$ of $7.94^{\circ}, 9.40^{\circ}, 10.10^{\circ}, 14.27^{\circ}, 16.11^{\circ}$, $21.25 .82^{\circ}, 26.78^{\circ}, 28.94^{\circ}$ and $38.70^{\circ}$. As for the PM-BVP-NS and BVP-NS, the typical crystal peaks of the of BVP were still appeared but with a little decreased in intensity. This further indicated that most of BVP in NS preparation remained in a crystalline form, while some of transferred to amorphous after wet grinding and spray drying, which was agreement with the PLM and DSC results. For the BVP-LP and BVP-PLC (1:2), the characteristic crystal peaks of BVP disappeared, indicating that BVP in BVP-LP and BVP-PLC (1:2) was highly dispersed in the form of molecules or amorphous morphology, which could validate with pervious conclusion.

\subsection{In Vitro Release Studies}

In vitro release study is a very important evidence to predict the in vivo absorption profile. As shown in Figure 9a, the total dissolution amount of the PM-BVP-NS was less than $7 \%$ until $120 \mathrm{~min}$, while BVP-NS exhibited higher and quicker cumulative release profile with the final release amount of $63.77 \pm 3.58 \%$, which was mainly attributed to the decrease in particle size to the nanometer range and the increased surface area [22].

The in vitro release profiles of BVP-LP and PM-BVP-LP were depicted in Figure 9b. The release of the physical mixture was very slow and the final amount in $2 \mathrm{~h}$ was less than $7 \%$. After preparing BVP into liposomes, the in vitro release was improved and extended with the final release amount reached $51.50 \pm 1.32 \%$, which confirmed that preparing BVP into liposomes could improve the drug release, to a certain extent.

Figure 9c showed the in vitro release profiles of PM-BVP-PLC and BVP-PLC. The dissolution behavior of the physical mixture was similar with that of PM-BVP-NS for about 7\%. Notably, compared to the PM-BVP-PLC, the BVP-PLC showed a dramatic enhancement of the rate and extent of dissolution, no matter which drug-lipid ratio. In specific, the dissolution behaviors of the BVP-PLC with drug-lipid ratios of 1:1 and 1:3 were similar, while the latter was slightly better than that of the former, with a cumulative release of $53.22 \pm 3.58 \%$ and $58.53 \pm 0.85 \%$, respectively. Obviously, the BVP-PLC with a drug-lipid ratio of 1:2 exhibited the most fast and complete dissolution behavior, with a cumulative release of $80.21 \pm 0.17 \%$ at $5 \mathrm{~min}$ and $100.65 \pm 0.72 \%$ at $120 \mathrm{~min}$. The reason was that the excessive phospholipids in the BVP-PLC with lower drug-lipid ratio (1:3) complexed BVP too tightly which hindered its dissolution. When it came to the BVP-PLC with higher drug-lipid ratio (1:1), phospholipids could not provide strong enough interaction force 
to complex BVP and maintain the stability of preparation, which resulted the destruction of PLC and the aggregation of BVP in dissolution media and further the compromised dissolution behavior.

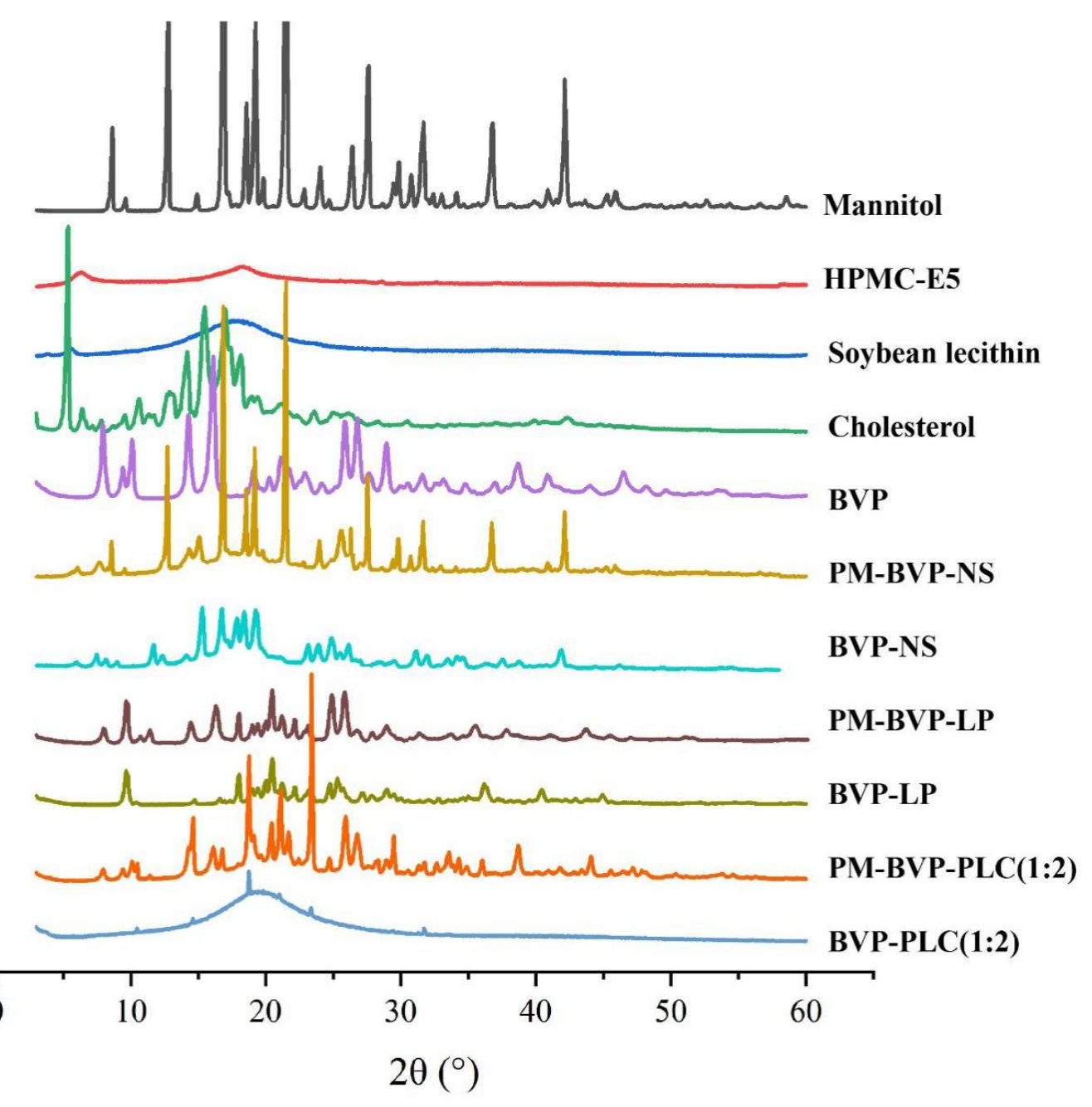

Figure 8. Powder X-ray diffraction (PXRD) pattern of mannitol, HPMC-E5, soybean lecithin, cholesterol, BVP, PM-BVP-NS, BVP-NS, PM-BVP-LP, BVP-LP, PM-BVP-PLC (1:2) and BVP-PLC (1:2).
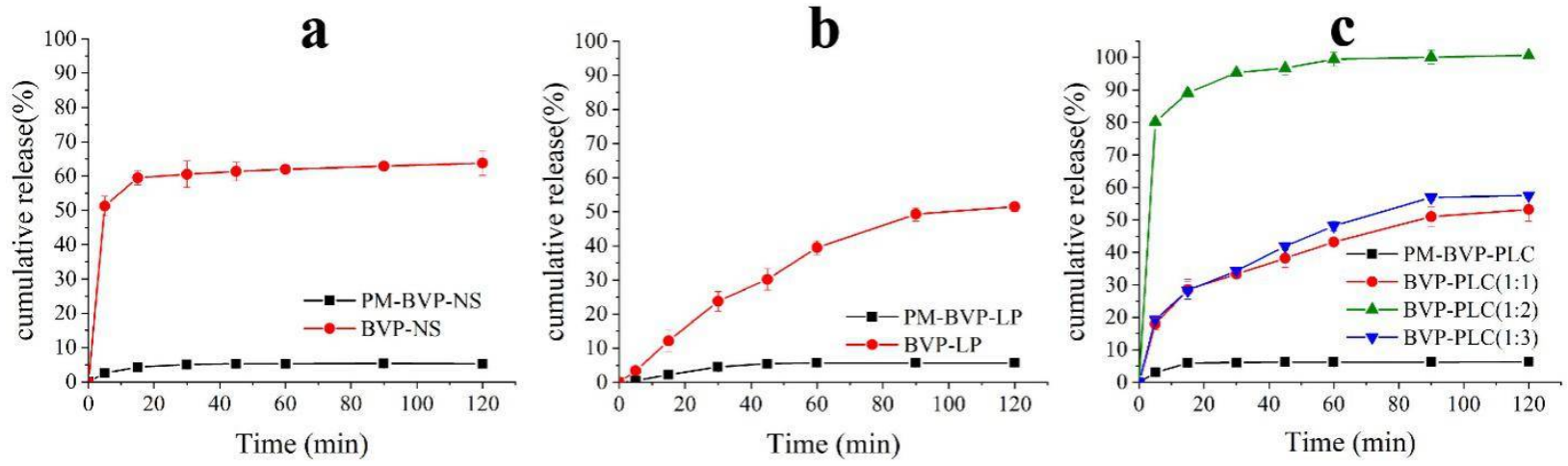

Figure 9. The cumulative release curves of BVP-NS (a), BVP-LP (b) and BVP-PLC (c). 


\subsection{Pharmacokinetics Study}

3.3.1. Pharmacokinetics Results of Different Preparations: BVP-NS, BVP-LP and BVP-PLC

The pharmacokinetic characteristics of BVP-NS, BVP-LP and BVP-LC (1:2) were investigated in male SD rats after single administration of $20 \mathrm{mg}$ BVP (i.g.). Figure 10 showed the concentration-time curve of different preparations and the specific pharmacokinetic parameters obtained from DAS software were summarized in Table 3. For BVP bulk drug, the plasma drug concentration reached the peak value $(101.70 \pm 27.45 \mu \mathrm{g} / \mathrm{L})$ at $3.60 \pm 0.89 \mathrm{~h}$ $\left(\mathrm{T}_{\max }\right)$ with the half life time $\left(\mathrm{t}_{1 / 2}\right)$ of $3.50 \pm 0.53 \mathrm{~h}$ and the $\mathrm{AUC}_{(0-\mathrm{t})}$ and $\mathrm{MRT}_{(0-\mathrm{t})}$ was $803.43 \pm 168.33 \mu \mathrm{g} / \mathrm{L} \cdot \mathrm{h}$ and $7.30 \pm 0.49 \mathrm{~h}$, respectively. The $\mathrm{T}_{\max }$ of BVP-NS $(2.00 \pm 0.00 \mathrm{~h})$ was shorter than that of BVP, while the $\mathrm{T}_{\max }$ of BVP-LP $(6.80 \pm 1.10 \mathrm{~h})$ and BVP-PLC $(1: 2)(12.00 \pm 0.00 \mathrm{~h})$ were both extended. Compared with BVP, all the three preparations showed different degrees of extending in vivo circulation time, with the $\mathrm{MRT}_{(0-\mathrm{t})}$ and $\mathrm{t}_{1 / 2 \mathrm{z}}$ of $6.70 \pm 0.41 \mathrm{~h}$ and $4.20 \pm 0.76 \mathrm{~h}$ for BVP-NS, $10.26 \pm 0.74 \mathrm{~h}$ and $8.89 \pm 1.40 \mathrm{~h}$ for BVP-LP, $10.79 \pm 0.25 \mathrm{~h}$ and $8.95 \pm 2.80 \mathrm{~h}$ for BVP-PLC (1:2), respectively. As for the maximum concentration, the BVP-NS possessed the highest $C_{\max }$ of $(444.06 \pm 54.10 \mu \mathrm{g} / \mathrm{L})$, the second for BVP-PLC $(291.20 \pm 43.08 \mu \mathrm{g} / \mathrm{L})$ and BVP-LP $(166.82 \pm 17.72 \mu \mathrm{g} / \mathrm{L})$ was the lowest one.

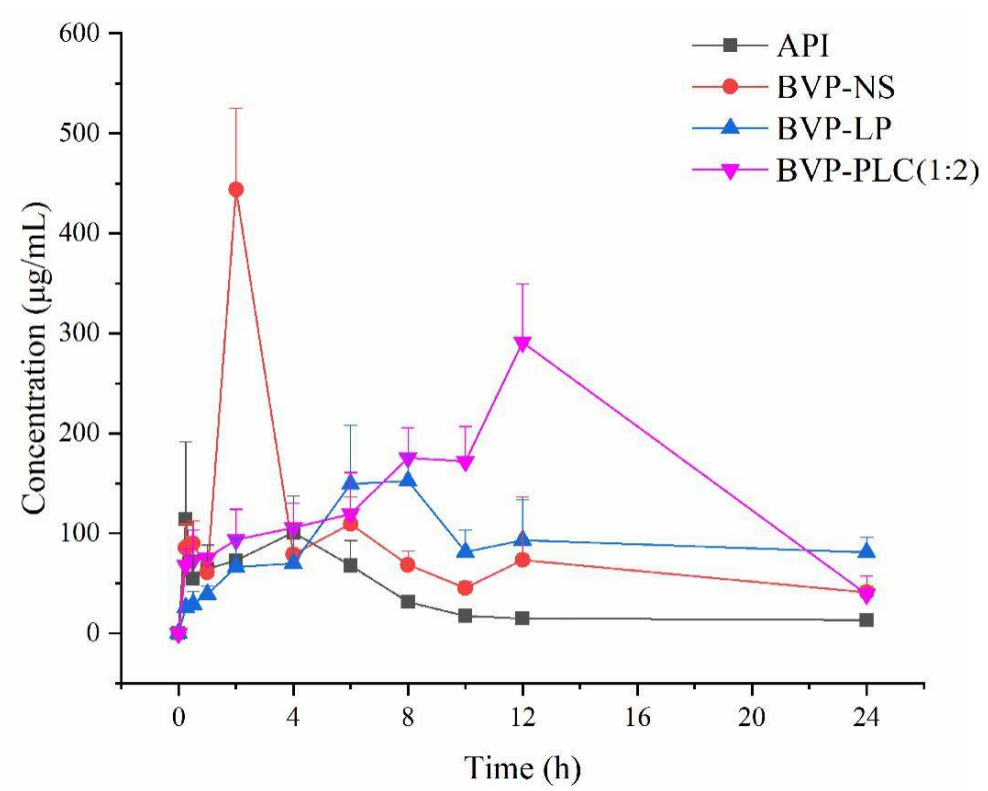

Figure 10. The plasma concentration-time curves of BVP, BVP-NS, BVP-LP and BVP-PLC (1:2) after single administration (i.g.) $(n=5)$.

Table 3. Pharmacokinetics parameters of BVP, nanosuspensions, liposomes and complexes (equivalent to BVP $20 \mathrm{mg} / \mathrm{kg}$ ).

\begin{tabular}{|c|c|c|c|c|c|}
\hline Parameter & Unit & BVP & BVP-NS & BVP-LP & BVP-PLC (1:2) \\
\hline $\mathrm{AUC}_{(0-\mathrm{t})}$ & $\mu \mathrm{g} / \mathrm{L} \cdot \mathrm{h}$ & $803.43 \pm 168.33$ & $1976.22 \pm 185.29 * *$ & $1908.26 \pm 316.62 * *$ & $3786.72 \pm 356.01$ ** \\
\hline $\mathrm{AUC}_{(0-\infty)}$ & $\mu \mathrm{g} / \mathrm{L} \cdot \mathrm{h}$ & $810.65 \pm 171.45$ & $2007.77 \pm 194.50 * *$ & $2422.41 \pm 491.91 * *$ & $4460.31 \pm 597.51^{* *}$ \\
\hline $\operatorname{MRT}_{(0-\mathrm{t})}$ & $\mathrm{h}$ & $7.30 \pm 0.49$ & $6.70 \pm 0.41$ & $10.26 \pm 0.74^{* *}$ & $10.79 \pm 0.25^{* *}$ \\
\hline $\operatorname{MRT}_{(0-\infty)}$ & $\mathrm{h}$ & $9.57 \pm 0.59$ & $9.41 \pm 1.07$ & $15.83 \pm 2.36^{* *}$ & $14.75 \pm 2.75^{*}$ \\
\hline$t_{1 / 2 z}$ & $\mathrm{~h}$ & $3.50 \pm 0.53$ & $4.20 \pm 0.76$ & $8.89 \pm 1.40^{* *}$ & $8.95 \pm 2.80^{* *}$ \\
\hline $\mathrm{T}_{\max }$ & $\mathrm{h}$ & $3.60 \pm 0.89$ & $2.00 \pm 0.00 *$ & $6.8 \pm 1.10^{* *}$ & $12 \pm 0$ ** \\
\hline $\mathrm{C}_{\max }$ & $\mu \mathrm{g} / \mathrm{L}$ & $101.70 \pm 27.45$ & $444.06 \pm 54.10^{* *}$ & $166.82 \pm 17.72 * *$ & $291.20 \pm 43.08 * *$ \\
\hline Relative Bioavailability & & - & $245.97 \%$ & $237.51 \%$ & $471.32 \%$ \\
\hline
\end{tabular}

All the data were presented in the form of mean $\pm \mathrm{SD}, n=5 .{ }^{*}$ indicates a statistically significant difference between BVP and BVP-NS, BVP-LP or BVP-PLC $(1: 2)(p<0.05) ;{ }^{* *}$ indicates $p<0.01$ vs. BVP.

As for BVP-NS, it could be seen clearly that the plasma concentration curve rapidly increased to the peak level $(444.06 \pm 54.10 \mu \mathrm{g} / \mathrm{L})$ within $2 \mathrm{~h}$ and diminished to a relatively low level $(153.14 \pm 35.69 .03 \mu \mathrm{g} / \mathrm{L})$ at $4 \mathrm{~h}$ indicated the rapid absorption and elimination of 
the BVP-NS in rats. This was highly consistent with the in vitro release behavior, where the most of drug released rapidly in the early stage. In general, the $\mathrm{AUC}_{(0-\mathrm{t})}$ of BVP-NS was $1976.22 \pm 185.29 \mu \mathrm{g} / \mathrm{L} \cdot \mathrm{h}$ which was 2.46 times of BVP. Moreover, mean residence time $\left(\mathrm{MRT}_{(0-\mathrm{t})}\right)$ of BVP-NS had no significant difference $(p>0.05)$ compared with BVP and the overall tendency of drug-time curve were also similar, which indicated the drug in BVP-NS was mainly existed in the original aggregated crystal form. It was worth noting that the $\mathrm{T}_{\max }$ of BVP-NS $(2.00 \pm 0.00)$ was shorter than BVP bulk drug $(3.60 \pm 0.89)$ and the $\mathrm{C}_{\max }$ was much higher, which might attribute to its smaller particle size and greater specific surface area and further the increased contact area with GIT and finally resulted faster absorption and higher oral bioavailability. In addition, a relatively small part of small insoluble particles of BVP-NS could be taken up via microfold (M) cells endocytosis [48], which might further enhance the oral bioavailability.

For the BVP-LP, the plasma drug concentration increased slowly within the first four hours, which still lower than that of BVP. However, it exhibited a relatively fast release at $6 \mathrm{~h}$ and reached the maximum concentration at $8 \mathrm{~h}$ and then entered in a slowly elimination phase. This characteristic might be related to the particular absorption behavior of liposomes after oral administration [23], whereby the liposomes could partially be destroyed following exposure to gastric acid, digestive enzymes and intestinal surfactants and resulted the release of free BVP. Therefore, the drug-time curve of BVP-LP before $4 \mathrm{~h}$ was mainly attributed to the released free BVP. The bilayer structure of liposomes was similar with the cell membrane and therefore liposomes that survived were able to be absorbed via the $\mathrm{M}$ cell-to-lymph pathway and epithelium endocytosis which conduced to the plasma drug concentration time curve after $4 \mathrm{~h}$. Most importantly, liposomes absorbed via the M-cell to lymphoid pathway could enter the blood circulation directly without passing through liver metabolism, which could further improve the absorption [23]. These particular absorption mechanisms endowed BVP-LP with extended absorption character and relatively higher oral bioavailability for about 2.38 times of BVP.

With regard to BVP-PLC (1:2), the plasma drug concentration continued to exhibited an upward tendency and reached the maximum value $(291.20 \pm 43.08 \mu \mathrm{g} / \mathrm{L})$ at $12 \mathrm{~h}$. Notably, BVP-PLC (1:2) possessed the supreme AUC $(0-\mathrm{t})(3786.72 \pm 356.01 \mu \mathrm{g} / \mathrm{L} \cdot \mathrm{h})$ and the relative bioavailability was 4.71 times of BVP, which mainly attributed to its unique absorption mechanism except for the epithelial endocytosis effect. BVP was negatively charged due to its polyphenol structure which ensured the formation of complex with positively charged phospholipid based on electrostatic interaction. In addition, based on previous characterization, there still existed the other form of interaction between BVP and phospholipid (Figure 11). These diverse and relatively strong interactions could maintain the integrity of BVP-PLC (1:2) in GIT and raised possibility for the following absorption process. In specific, BVP-PLC (1:2) could be hydrolyzed to fatty acid and BVP-monoacylglycerol in the presence of phospholipase (especially phospholipase A2) in GIT [49], which could further combine with bile salts and then being absorbed by epithelial cell. The excretion of bile salts is regulated by a hormone called cholecystokinin (CCK), which is sensitive to the concentration of fatty acid. Interestingly, the hydrolysis of BVP-PLC (1:2) could increase the concentration of free fatty acid, which could trigger CCK release and further regulate bile excretion, finally a positive feedback loop kicked in. Once the BVP-monoacylglycerol complex was internalized by epithelial cell in GIT, it would be processed by the enzymes in the smooth endoplasmic reticulum to BVP-diacyl phospholipids. After that, in Golgi apparatus, apolipoprotein B-48 would be integrated to form chylomicrons. Then, BVP loaded chylomicron excreted from epithelial cell through exocytosis and entered in lymphatic circulation, which allowed the BVP-PLC enter in systemic circulation directly and bypass the liver metabolism. 


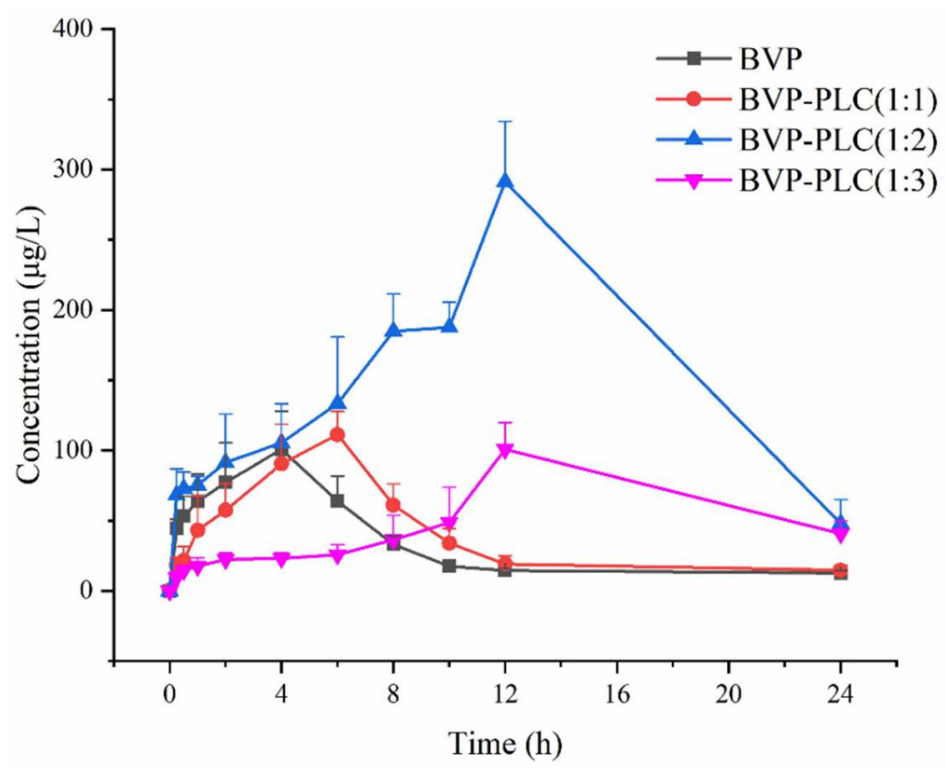

Figure 11. The plasma concentration-time curves of BVP and BVP-PLC with different drug-lipid ratios after single administration (i.g.).

To sum up, BVP-PLC (1:2) was the most promising preparation of the three to address the low bioavailability of BVP. For the BVP-NS, although it could improve the oral bioavailability of the drug and had highest $C_{\max }$ of the three, the fast systemic metabolism would hinder its further application. Therefore, the main task of BVP-NS was to extend the release of drug and prolong the circulation time in vivo. Some researchers have performed relevant studies in this field, Peng Ji et al. modified curcumin nanocrystals with hyaluronic acid, which raised the blood elimination half-life from $14.66 \pm 6.58 \mathrm{~h}$ to $53.06 \pm 18.21 \mathrm{~h} \mathrm{[50]}$ Although BVP-LP could significantly extend the mean retention time and half-life time, the relative bioavailability was not enhanced greatly compared with BVP-NS. Phospholipid complexes could both significantly increase the oral bioavailability and prolong the half-life time of the drug. Notably, in vitro release study showed that the drug-lipid ratio had remarkable influence on its dissolution behavior. Therefore, the pharmacokinetics of the BVP-PLC with different drug-lipid ratios were studied.

\subsubsection{Pharmacokinetic Results of BVP-PLC with Different Drug-Lipid Ratios}

According to the in vitro release results, the drug-lipid ratios had significant influence on dissolution behavior, therefore the corresponding pharmacokinetic study was also carried out and the concentration-time curve and pharmacokinetic parameters were shown in Figure 11 and Table 4, respectively. For the BVP-PLC (1:1), almost all pharmacokinetic parameters $\left(\mathrm{AUC}_{(0-\mathrm{t})}, \mathrm{AUC}_{(0-\infty)}, \mathrm{MRT}_{(0-\infty)}, \mathrm{t}_{1 / 2}, \mathrm{C}_{\max }\right)$ showed no significant difference compared with BVP except for the MRT $(0-t)(8.03 \pm 0.31 \mathrm{~h})$ and $\mathrm{T}_{\max }(5.60 \pm 0.89 \mathrm{~h})$ with a little prolonged and the relative bioavailability was $118.06 \%$ which was also not greatly improved. The BVP-PLC with 1:2 drug-lipid ratio possessed the highest relative bioavailability of BVP $(471.32 \%)$ and the maximum $\mathrm{T}_{\max }(12.00 \pm 0.00 \mathrm{~h})$ and $\mathrm{C}_{\max }(291.20$ $\pm 43.08 \mu \mathrm{g} / \mathrm{L})$, while with the moderate $\mathrm{MRT}_{(0-\mathrm{t})}(10.79 \pm 0.25 \mathrm{~h})$ and $\mathrm{t}_{1 / 2}(8.95 \pm 2.80 \mathrm{~h})$. With regard to the BVP-PLC with drug-lipid ratio of 1:3, its $\mathrm{AUC}_{(0-\mathrm{t})}$ was significantly enhanced for about 1.57 times of BVP and possessed the same $T_{\max }$ with BVP-PLC (1:2) and the longest $\mathrm{MRT}_{(0-\mathrm{t})}(12.99 \pm 0.71 \mathrm{~h})$ and $\mathrm{t}_{1 / 2}(17.67 \pm 3.63 \mathrm{~h})$, while the smallest $\mathrm{C}_{\max }(100.95 \pm 19.00 \mu \mathrm{g} / \mathrm{L})$ among the three. Therefore, it could be concluded that the drug-lipid ratio indeed had significantly influence on the in vivo process. In specific, the lower drug-lipid ratio was conducive to the longer circulation time in vivo, while the relative bioavailability possessed an entirely different tendency. As could be seen in Table 4, BVP-PLC (1:2) had the highest relative bioavailability and second for BVP-PLC (1:3), the last for BVP-PLC (1:1). 
Table 4. Pharmacokinetics parameters of BVP and BVP-PLC with different drug-lipid ratios (equivalent to BVP $20 \mathrm{mg} / \mathrm{kg}$ ).

\begin{tabular}{|c|c|c|c|c|c|}
\hline Parameter & Unit & BVP & 1: 1 & 1: 2 & 1: 3 \\
\hline $\mathrm{AUC}_{(0-\mathrm{t})}$ & $\mu \mathrm{g} / \mathrm{L} \cdot \mathrm{h}$ & $803.43 \pm 168.33$ & $948.50 \pm 70.46$ & $3786.72 \pm 356.01^{* *}$ & $1261.41 \pm 124.99 * *$ \\
\hline $\mathrm{AUC}_{(0-\infty)}$ & $\mu \mathrm{g} / \mathrm{L} \cdot \mathrm{h}$ & $810.65 \pm 171.45$ & $1002.07 \pm 90.48$ & $4460.31 \pm 597.51^{* *}$ & $2327.07 \pm 321.38^{* *}$ \\
\hline $\operatorname{MRT}_{(0-\mathrm{t})}$ & $\mathrm{h}$ & $7.30 \pm 0.49$ & $8.03 \pm 0.31 *$ & $10.79 \pm 0.25^{* *}$ & $12.99 \pm 0.71$ ** \\
\hline $\operatorname{MRT}_{(0-\infty)}$ & $\mathrm{h}$ & $9.57 \pm 0.59$ & $10.47 \pm 0.83$ & $14.75 \pm 2.75^{*}$ & $29.72 \pm 6.56^{* *}$ \\
\hline$t_{1 / 2 z}$ & $\mathrm{~h}$ & $3.50 \pm 0.53$ & $4.69 \pm 2.82$ & $8.95 \pm 2.80^{* *}$ & $17.67 \pm 3.63^{* *}$ \\
\hline $\mathrm{T}_{\max }$ & $\mathrm{h}$ & $3.60 \pm 0.89$ & $5.60 \pm 0.89^{* *}$ & $12.00 \pm 0.00 * *$ & $12.00 \pm 0.00 * *$ \\
\hline $\mathrm{C}_{\max }$ & $\mu \mathrm{g} / \mathrm{L}$ & $101.70 \pm 27.45$ & $117.22 \pm 17.73$ & $291.20 \pm 43.08^{* *}$ & $100.95 \pm 19.00$ \\
\hline Relative Bioavailability & & - & $118.06 \%$ & $471.32 \%$ & $157.00 \%$ \\
\hline
\end{tabular}

All the data were presented in the form of mean $\pm \mathrm{SD}, n=5$. * indicates a statistically significant difference between BVP and BVP-PLC $(p<0.05) ;{ }^{* *}$ indicates $p<0.01$ vs. BVP.

This unusual tendency might attribute to the distinct interaction type and strength between drug and phospholipids in the BVP-PLC with different drug-lipid ratios, which further resulted in disparate preparation stability, drug absorption in GIT and release behavior in vivo. Through molecular simulation, it was found that there are mainly two kinds of forces (hydrogen bond and electrostatic interaction). For BVP-PLC (1:1), the molar ratio of drug to phospholipid was about 2:1 or even higher. So, the relatively less phospholipid molecule could not provide strong enough interaction forces to complex BVP and maintain the stability of preparation, which resulted its structure was very easy to be destroyed in GIT and the aggregation of drug, therefore its pharmacokinetic behavior was similar to BVP bulk drug and the $\mathrm{AUC}_{(0-\mathrm{t})}$ had no significant difference. When the drug-lipid ratio raised to 1:2, the molar ratio of phospholipid and drug was about 1:1. Based on the previous results, this ratio might be the most satisfactory prescription, which could provide moderate interaction to exactly balance the drug loading and drug release. On the one hand, the BVP-PLC at this drug-lipid ratio could maintain the integrity of the complex structure in GIT, which ensured the relatively higher absorption. On the other hand, this suitable interaction could also make the drug release more complete and thereby the oral bioavailability (471.32\%) was improved greatly. As for drug-lipid ratio of 1:3 or even lower, the phospholipid in preparation was so overwhelmed that the encapsulation forces were too tightly, which hindered the release of BVP from the preparation and leading to a relatively lower relative bioavailability (157.00\%). Therefore, it could be concluded that the drug-lipid ratio played a very important role in oral bioavailability of BVP-PLC, the more suitable drug-lipid ratio, the higher oral bioavailability.

\section{Conclusions}

The absorption process, in vivo stability and release profile are all very important factor to the oral bioavailability of BVP. In this study, three preparations, BVP-NS, BVP-LP and BVP-PLC were investigated. The pharmaceutical study indicated that the particle size of the three preparations was in the range of $200-300 \mathrm{~nm}$ and the zeta potential was all negative. The encapsulation efficiency of BVP-LP was $76.41 \pm 5.61 \%$ and the complexation efficiency of BVP-PLC (1:2) was $72.90 \pm 3.21 \%$. PLM, PXRD and DSC results suggested that BVP was presented as crystal in nanosuspension, while as non-crystal form in liposome and phospholipid complex. FI-IR experiment demonstrated that the $-\mathrm{OH}$ and $-\mathrm{COO}^{-}$group in BVP could interact with phospholipid molecule during phospholipid complex formation, rather than nanosuspension and liposome. Molecular simulation further confirmed that hydrogen bond and electrostatic interaction were the two main kinds interactions. In vitro release results manifested that BVP-PLC (1:2) showed the highest cumulative release amount of $100.65 \pm 0.72 \%$, followed by BVP-NS $(63.77 \pm 3.58 \%)$ and BVP-LP (51.50 $\pm 1.32 \%)$. In vivo pharmacokinetic study verified that BVP-NS, BVP-LP and BVP-PLC all shown significantly higher oral bioavailability compared with BVP, with the relative bioavailability of $245.97 \%, 237.51 \%$ and $471.32 \%$, respectively. In addition, BVPLP and BVP-PLC exhibited a distinctly extended-release profile with the $\mathrm{MRT}_{(0-\mathrm{t})}$ of $10.26 \pm$ 0.74 and $10.79 \pm 0.25$, compared with BVP $(7.30 \pm 0.49)$. Furthermore, the drug-lipid ratio had significant influence on the pharmacokinetic parameters of BVP-PLC, in which the 
preparation with drug-lipid ratio of 1:2 had the highest relative bioavailability (471.32\%). As the higher drug-lipid ratio (1:1) could not provide strong enough force to maintain the stability of the preparation and the lower drug-lipid ratio (1:3) impacted the dissociation of BVP from the preparation. Therefore, phospholipid complexes with appropriate drug-lipid ratio provide an effective strategy for improving the oral bioavailability of drugs which have low solubility both in water and lipid.

Author Contributions: Z.S.: Conceptualization, Investigation, Methodology, writing—original draft, Writing—review \& editing. J.Y.: Investigation, Methodology, Writing—review \& editing. P.X.: Methodology, Investigation. J.C.: Methodology, Investigation. J.G.: Formal analysis, Visualization. Y.W.: Formal analysis, Visualization. Y.Z.: Formal analysis, Visualization. T.Y.: Formal analysis, Visualization. X.T.: Resource, Conceptualization, Project administration, Supervision, Writingreview \& editing. H.H.: Conceptualization, Visualization, Project administration, Supervision, Writing-review \& editing. All authors have read and agreed to the published version of the manuscript.

Funding: This research was funded by the National Mega-project for Innovative Drugs [No. 2019ZX09721001], Program for Liaoning Innovative Talents in University (2012520007), National Natural Science Foundation of China (81673378), Liaoning Revitalization Talents Program [XLYC1908031]. National Key R\&D Program of China (2020YFE0201700).

Institutional Review Board Statement: All the animal research was approved by the Committee of Ethics of Animal Experimentation of Shenyang Pharmaceutical University and carried out in accordance with the guidelines of this committee (SYPU-IACUC-C2019-7-3-204).

Informed Consent Statement: Not applicable.

Data Availability Statement: The data presented in this study are available on request from the corresponding author. The data are not publicly available due to privacy.

Acknowledgments: We were grateful for Amanda Pearce to correct the manuscript.

Conflicts of Interest: The authors declare no conflict of interest.

\section{References}

1. Wang, X.; Xia, H.; Liu, Y.; Qiu, F.; Di, X. Simultaneous determination of three glucuronide conjugates of scutellarein in rat plasma by LC-MS/MS for pharmacokinetic study of breviscapine. J. Chromatogr. B Anal. Technol. Biomed. Life Sci. 2014, 965, 79-84. [CrossRef]

2. Zhao, J.; Yang, J.; Xie, Y. Improvement strategies for the oral bioavailability of poorly water-soluble flavonoids: An overview. Int. J. Pharm. 2019, 570, 118642. [CrossRef]

3. Wang, M.; Zhang, W.-B.; Song, J.-L.; Luan, Y.; Jin, C.-Y. Effect of Breviscapine on Recovery of Viable Myocardium and Left Ventricular Remodeling in Chronic Total Occlusion Patients After Revascularization: Rationale and Design for a Randomized Controlled Trial. Med. Sci. Monit. 2018, 24, 4602-4609. [CrossRef]

4. Guo, C.; Zhu, Y.; Weng, Y.; Wang, S.; Guan, Y.; Wei, G.; Yin, Y.; Xi, M.; Wen, A. Therapeutic time window and underlying therapeutic mechanism of breviscapine injection against cerebral ischemia/reperfusion injury in rats. J. Ethnopharmacol. 2014, 151, 660-666. [CrossRef]

5. Li, X.L.; Li, Y.Q.; Yan, W.M.; Li, H.Y.; Xu, H.; Zheng, X.X.; Guo, D.W.; Tang, L.K. A study of the cardioprotective effect of breviscapine during hypoxia of cardiomyocytes in vitro and during myocardial infarction in vivo. Planta Med. 2004, 70, 1039-1044. [CrossRef]

6. Wang, J.; Ji, S.-Y.; Liu, S.-Z.; Jing, R.; Lou, W.-J. Cardioprotective effect of breviscapine: Inhibition of apoptosis in H9c2 cardiomyocytes via the PI3K/Akt/eNOS pathway following simulated ischemia/reperfusion injury. Pharmazie 2015, 70, 593-597.

7. Zhang, P.; Guo, T.; He, H.; Yang, L.; Deng, Y. Breviscapine confers a neuroprotective efficacy against transient focal cerebral ischemia by attenuating neuronal and astrocytic autophagy in the penumbra. Biomed. Pharmacother. 2017, 90, 69-76.

8. Jiang, L.; Xia, Q.J.; Dong, X.J.; Hu, Y.; Chen, Z.W.; Chen, K.; Wang, K.H.; Liu, J.; Wang, T.H. Neuroprotective effect of breviscapine on traumatic brain injury in rats associated with the inhibition of GSK3beta signaling pathway. Brain Res. 2017, 1660, 1-9. [CrossRef]

9. Wang, L.; Ma, Q. Clinical benefits and pharmacology of scutellarin: A comprehensive review. Pharmacol. Ther. 2018, 190, 105-127. [CrossRef]

10. Xiong, F.; Wang, H.; Geng, K.-K.; Gu, N.; Zhu, J.-B. Optimized Preparation, Characterization and Biodistribution in Heart of Breviscapine Lipid Emulsion. Chem. Pharm. Bull. 2010, 58, 1455-1460. [CrossRef] 
11. Yang, X.; Miao, X.; Cao, F.; Li, S.; Ai, N.; Chang, Q.; Lee, S.M.Y.; Zheng, Y. Nanosuspension Development of Scutellarein as an Active and Rapid Orally Absorbed Precursor of its BCS Class IV Glycoside Scutellarin. J. Pharm. Sci. 2014, 103, 3576-3584. [CrossRef]

12. Cui, M.-Y.; Tian, C.-C.; Ju, A.-X.; Zhang, C.-T.; Li, Q.-H. Pharmacokinetic interaction between scutellarin and valsartan in rats. Acta Pharm. Sin. 2013, 48, 541-546.

13. Hao, X.H.; Cheng, G.; Yu, J.; He, Y.X.; An, F.; Sun, J.; Cui, F.D. Study on the role of hepatic first-pass elimination in the low oral bioavailability of scutellarin in rats. Pharmazie 2005, 60, 477-478.

14. Xing, J.-F.; You, H.-S.; Dong, Y.-L.; Lu, J.; Chen, S.-Y.; Zhu, H.-F.; Dong, Q.; Wang, M.-Y.; Dong, W.-H. Metabolic and pharmacokinetic studies of scutellarin in rat plasma, urine, and feces. Acta Pharmacol. Sin. 2011, 32, 655-663. [CrossRef]

15. Chen, X.; Cui, L.; Duan, X.; Ma, B.; Zhong, D. Pharmacokinetics and metabolism of the flavonoid scutellarin in humans after a single oral administration. Drug Metab. Dispos. 2006, 34, 1345-1352. [CrossRef] [PubMed]

16. Xiong, F.; Wang, H.; Cheng, J.; Zhu, J.B. Determination of scutellarin in mouse plasma and different tissues by high-performance liquid chromatography. J. Chromatogr. B Anal. Technol. Biomed. Life Sci. 2006, 835, 114-118. [CrossRef]

17. Huang, J.M.; Weng, W.Y.; Huang, X.B.; Ji, Y.H.; Chen, E. Pharmacokinetics of scutellarin and its aglycone conjugated metabolites in rats. Eur. J. Drug Metab. Pharmacokinet. 2005, 30, 165-170. [CrossRef]

18. Zhou, Y.; Ning, Q.; Yu, D.-N.; Li, W.-G.; Deng, J. Improved oral bioavailability of breviscapine via a Pluronic P85-modified liposomal delivery system. J. Pharm. Pharmacol. 2014, 66, 903-911. [CrossRef]

19. Xie, J.; Luo, Y.; Chen, Y.; Ma, Y.; Yue, P.; Yang, M. Novel breviscapine nanocrystals modified by panax notoginseng saponins for enhancing bioavailability and synergistic anti-platelet aggregation effect. Colloids Surf. B Biointerfaces 2019, 175, 333-342.

20. Ma, Y.; Li, H.; Guan, S. Enhancement of the oral bioavailability of breviscapine by nanoemulsions drug delivery system. Drug Dev. Ind. Pharm. 2015, 41, 177-182. [CrossRef]

21. Samir, A.; Elgamal, B.M.; Gabr, H.; Sabaawy, H.E. Nanotechnology applications in hematological malignancies. Oncol. Rep. 2015, 34, 1097-1105. [CrossRef] [PubMed]

22. Liu, Q.; Mai, Y.; Gu, X.; Zhao, Y.; Di, X.; Ma, X.; Yang, J. A wet-milling method for the preparation of cilnidipine nanosuspension with enhanced dissolution and oral bioavailability. J. Drug Deliv. Sci. Technol. 2020, 55, 101371. [CrossRef]

23. He, H.; Lu, Y.; Qi, J.; Zhu, Q.; Chen, Z.; Wu, W. Adapting liposomes for oral drug delivery. Acta Pharm. Sin. B 2019, 9, 36-48. [CrossRef]

24. Bao, R.; Wang, Q.-L.; Li, R.; Adu-Frimpong, M.; Toreniyazov, E.; Ji, H.; Xu, X.-M.; Yu, J.-N. Improved oral bioavailability and target delivery of 6-shogaol via vitamin E TPGS-modified liposomes: Preparation, in-vitro and in-vivo characterizations. J. Drug Deliv. Sci. Technol. 2020, 59, 101842. [CrossRef]

25. Guo, Y.; Shen, L.-X.; Lu, Y.-F.; Li, H.-Y.; Min, K.; Li, L.-F.; Yu, C.-Y.; Zheng, X. Preparation of Rutin-liposome Drug Delivery Systems and Evaluation on Their in vitro Antioxidant Activity. Chin. Herb. Med. 2016, 8, 371-375. [CrossRef]

26. Toniazzo, T.; Peres, M.S.; Ramos, A.P.; Pinho, S.C. Encapsulation of quercetin in liposomes by ethanol injection and physicochemical characterization of dispersions and lyophilized vesicles. Food Biosci. 2017, 19, 17-25. [CrossRef]

27. Silva-Weiss, A.; Quilaqueo, M.; Venegas, O.; Ahumada, M.; Silva, W.; Osorio, F.; Gimenez, B. Design of dipalmitoyl lecithin liposomes loaded with quercetin and rutin and their release kinetics from carboxymethyl cellulose edible films. J. Food Eng. 2018, 224, 165-173. [CrossRef]

28. Liu, W.; Kong, Y.; Ye, A.; Shen, P.; Dong, L.; Xu, X.; Hou, Y.; Wang, Y.; Jin, Y.; Han, J. Preparation, formation mechanism and in vitro dynamic digestion behavior of quercetin-loaded liposomes in hydrogels. Food Hydrocoll. 2020, 104, 105743. [CrossRef]

29. Hao, J.; Guo, B.; Yu, S.; Zhang, W.; Zhang, D.; Wang, J.; Wang, Y. Encapsulation of the flavonoid quercetin with chitosan-coated nano-liposomes. LWT Food Sci. Technol. 2017, 85, 37-44. [CrossRef]

30. Kumar, N.; Rai, A.; Reddy, N.D.; Raj, P.V.; Jain, P.; Deshpande, P.; Mathew, G.; Kutty, N.G.; Udupa, N.; Rao, C.M. Silymarin liposomes improves oral bioavailability of silybin besides targeting hepatocytes, and immune cells. Pharmacol. Rep. 2014, 66, 788-798. [CrossRef]

31. El-Samaligy, M.S.; Afifi, N.N.; Mahmoud, E.A. Evaluation of hybrid liposomes-encapsulated silymarin regarding physical stability and in vivo performance. Int. J. Pharm. 2006, 319, 121-129. [CrossRef] [PubMed]

32. Zhao, X.; Shi, C.; Zhou, X.; Lin, T.; Gong, Y.; Yin, M.; Fan, L.; Wang, W.; Fang, J. Preparation of a nanoscale dihydromyricetinphospholipid complex to improve the bioavailability: In vitro and in vivo evaluations. Eur. J. Pharm. Sci. 2019, 138, 104994. [CrossRef] [PubMed]

33. Lu, M.; Qiu, Q.; Luo, X.; Liu, X.; Sun, J.; Wang, C.; Lin, X.; Deng, Y.; Song, Y. Phyto-phospholipid complexes (phytosomes): A novel strategy to improve the bioavailability of active constituents. Asian J. Pharm. Sci. 2019, 14, 265-274. [CrossRef]

34. Zhang, K.; Zhang, M.; Liu, Z.; Zhang, Y.; Gu, L.; Hu, G.; Chen, X.; Jia, J. Development of quercetin-phospholipid complex to improve the bioavailability and protection effects against carbon tetrachloride-induced hepatotoxicity in SD rats. Fitoterapia 2016 113, 102-109. [CrossRef] [PubMed]

35. Zhang, K.; Gu, L.; Chen, J.; Zhang, Y.; Jiang, Y.; Zhao, L.; Bi, K.; Chen, X. Preparation and evaluation of kaempferol-phospholipid complex for pharmacokinetics and bioavailability in SD rats. J. Pharm. Biomed. Anal. 2015, 114, 168-175. [CrossRef]

36. Chi, C.; Zhang, C.; Liu, Y.; Nie, H.; Zhou, J.; Ding, Y. Phytosome-nanosuspensions for silybin-phospholipid complex with increased bioavailability and hepatoprotection efficacy. Eur. J. Pharm. Sci. 2020, 144, 105212. [CrossRef] [PubMed] 
37. Maiti, K.; Mukherjee, K.; Gantait, A.; Saha, B.P.; Mukherjee, P.K. Curcumin-phospholipid complex: Preparation, therapeutic evaluation and pharmacokinetic study in rats. Int. J. Pharm. 2007, 330, 155-163. [CrossRef]

38. Jena, S.K.; Singh, C.; Dora, C.P.; Suresh, S. Development of tamoxifen-phospholipid complex: Novel approach for improving solubility and bioavailability. Int. J. Pharm. 2014, 473, 1-9. [CrossRef]

39. Li, M.; Azad, M.; Davé, R.; Bilgili, E. Nanomilling of Drugs for Bioavailability Enhancement: A Holistic Formulation-Process Perspective. Pharmaceutics 2016, 8, 17. [CrossRef]

40. Chu, C.; Tong, S.S.; Xu, Y.; Wang, L.; Fu, M.; Ge, Y.R.; Yu, J.N.; Xu, X.M. Proliposomes for oral delivery of dehydrosilymarin: Preparation and evaluation in vitro and invivo. Acta Pharmacol. Sin. 2011, 32, 973-980. [CrossRef]

41. Chu, J.; Zou, C.; Li, C.; Zhang, J.; Zhao, Y.; Xu, M.; Wu, T.; Ju, W. Determination of scutellarein in human plasma by enzymatic hydrolysis and liquid chromatograph-triple quadrupole tandem mass spectrometer analysis: Its use in determining the bioequivalence of scutellarin in Chinese volunteers. Eur. J. Integr. Med. 2016, 8, 519-525. [CrossRef]

42. Bilgili, E.; Rahman, M.; Palacios, D.; Arevalo, F. Impact of polymers on the aggregation of wet-milled itraconazole particles and their dissolution from spray-dried nanocomposites. Adv. Powder Technol. 2018, 29, 2941-2956. [CrossRef]

43. Koradia, K.D.; Sheth, N.R.; Koradia, H.D.; Dabhi, M.R. Ziprasidone nanocrystals by wet media milling followed by spray drying and lyophilization: Formulation and process parameter optimization. J. Drug Deliv. Sci. Technol. 2018, 43, 73-84. [CrossRef]

44. Tan, Q.; Liu, S.; Chen, X.; Wu, M.; Wang, H.; Yin, H.; He, D.; Xiong, H.; Zhang, J. Design and Evaluation of a Novel EvodiaminePhospholipid Complex for Improved Oral Bioavailability. AAPS PharmSciTech 2012, 13, 534-547. [CrossRef]

45. Mazumder, A.; Dwivedi, A.; Preez, J.L.D.; Plessis, J.D. In vitro wound healing and cytotoxic effects of sinigrin-phytosome complex. Int. J. Pharm. 2016, 498, 283-293. [CrossRef]

46. Patel, P.J.; Gajera, B.Y.; Dave, R.H. A quality-by-design study to develop Nifedipine nanosuspension: Examining the relative impact of formulation variables, wet media milling process parameters and excipient variability on drug product quality attributes. Drug Dev. Ind. Pharm. 2018, 44, 1942-1952. [CrossRef] [PubMed]

47. Freag, M.S.; Elnaggar, Y.; Abdallah, O.Y. Lyophilized phytosomal nanocarriers as platforms for enhanced diosmin delivery: Optimization and ex vivo permeation. Int. J. Nanomed. 2013, 8, 2385-2397.

48. da Silva, A.B.; Miniter, M.; Thom, W.; Hewitt, R.E.; Wills, J.; Jugdaohsingh, R.; Powell, J.J. Gastrointestinal Absorption and Toxicity of Nanoparticles and Microparticles: Myth, Reality and Pitfalls explored through Titanium Dioxide. Curr. Opin. Toxicol. 2020, 19, 112-120. [CrossRef]

49. Kuche, K.; Bhargavi, N.; Parkash, C.; Jain, S. Drug-Phospholipid Complex-A Go Through Strategy for Enhanced Oral Bioavailability. AAPS PharmSciTech 2019, 20, 43. [CrossRef]

50. Ji, P.; Wang, L.; Chen, Y.; Wang, S.; Wu, Z.; Qi, X. Hyaluronic acid hydrophilic surface rehabilitating curcumin nanocrystals for targeted breast cancer treatment with prolonged biodistribution. Biomater. Sci. 2020, 8, 462-472. [CrossRef] 